\title{
Successive Wyner-Ziv Coding for the Binary CEO Problem under Logarithmic Loss
}

\author{
Mahdi Nangir, Reza Asvadi, Member, IEEE, Jun Chen, Senior Member, IEEE, \\ Mahmoud Ahmadian-Attari, and Tad Matsumoto, Fellow, IEEE
}

\begin{abstract}
The L-link binary Chief Executive Officer (CEO) problem under logarithmic loss is investigated in this paper. A quantization splitting technique is applied to convert the problem under consideration to a $(2 L-1)$-step successive Wyner-Ziv (WZ) problem, for which a practical coding scheme is proposed. In the proposed scheme, Low-Density Generator-Matrix (LDGM) codes are used for binary quantization while Low-Density Parity-Check (LDPC) codes are used for syndrome generation; the decoder performs successive decoding based on the received syndromes and produces a soft reconstruction of the remote source. The simulation results indicate that the rate-distortion performance of the proposed scheme can approach the theoretical inner bound based on binary-symmetric test-channel models.
\end{abstract}

Index Terms - Binary CEO problem, binary quantization, successive decoding, syndrome decoding, Wyner-Ziv problem, quantization splitting, logarithmic loss.

\section{INTRODUCTION}

Multiterminal source coding is an important subject of network information theory. Research on this subject has yielded insights and techniques that are useful for a wide range of applications, including, among other things, cooperative communications [2], distributed storage [3], and sensor networks [4]. A particular formulation of multiterminal source coding, known as the Chief Executive Officer (CEO) problem, has received significant attention [5]. In this problem, there are $L$ encoders (also called agents), which observe independently corrupted versions

M. Nangir was with the Faculty of Electrical Engineering, K.N. Toosi University of Technology, Tehran, Iran. He is now with the Faculty of Electrical and Computer Engineering, University of Tabriz, Tabriz, Iran (e-mail: nangir@tabrizu.ac.ir).

R. Asvadi is with the Faculty of Electrical Engineering, Shahid Beheshti University, Tehran, Iran (corresponding author, e-mail: r asvadi@sbu.ac.ir).

J. Chen is with the Department of Electrical and Computer Engineering, McMaster University, Hamilton, ON, Canada (e-mail: junchen@mail.ece.mcmaster.ca).

M. Ahmadian-Attari is with the Faculty of Electrical Engineering K.N. Toosi University of Technology, Tehran, Iran (e-mail: mahmoud@eetd.kntu.ac.ir).

T. Matsumoto is with the school of Information Science, Japan Advanced Institute of Science and Technology, Ishikawa 923-1292, Japan, and also with the Centre for Wireless Communications, University of Oulu, 90014 Oulu, Finland (e-mail: matumoto@jaist.ac.jp).

This paper was presented in part at the 29th Biennial Symposium on Communications (BSC 2018), Toronto, Canada [1].

This work was supported in part by the project 7th Framework Program (FP7) Links-on-the-fly Technology for Robust, Efficient and Smart Communication in Unpredictable Environments (RESCUE). of a source; these encoders compress their respective observations and forward the compressed data separately to a central decoder (also called CEO), which then produces a (lossy) reconstruction of the target source.

The quadratic Gaussian setting of the CEO problem has been studied extensively, for which the rate-distortion region is characterized completely in the scalar case and partially in the vector case. Extending these results beyond the quadratic Gaussian setting turns out to be highly nontrivial; there are some results in [6]-[8]. Indeed, even for many seemingly simple sources and distortion measures, the understanding of the relevant information-theoretic limits is rather limited. A remarkable exception is a somewhat under-appreciated distortion measure called logarithmic loss (log-loss). As shown by Courtade and Weissman [9], the rate-distortion region of the CEO problem under log-loss admits a single-letter characterization for arbitrary finite-alphabet sources and noisy observations. Different from the conventional distortion measures which are typically imposed on "hard" reconstructions defined over the given source alphabet, log-loss is tailored to "soft" reconstructions in the form of probability distributions. Specifically, in the context of the CEO problem, the most favorable "soft" reconstruction is essentially the a posteriori distribution of the source given the compressed data received from the encoders (which is a sufficient statistic); it is more informative than its "hard" counterparts and more suitable for many downstream statistical inference tasks.

Recent years have seen significant interests in a new paradigm of wireless communications called Cloud-Radio Access Network (C-RAN). It has been recognized that the information-theoretic and coding-theoretic aspect of CRAN is closely related to that of the CEO problem under log-loss [10]. This intriguing connection greatly enriches the implication of the latter problem and provides further motivations for the relevant research.

A main contribution of the present paper is a practical coding scheme for the CEO problem under log-loss. We adopt a hierarchical approach by decomposing the CEO problem into a set of simpler problems upon which the existing coding techniques can be directly brought to bear and then combining these small pieces to find the solution to the original problem. Two most basic problems in information theory are point-to-point channel coding and (lossy) source coding (also known as quantization). It is well known that the fundamental limits of these 
two problems can be approached using graph-based codes (e.g., Low-Density Parity-Check (LDPC) codes for channel coding [11] and Low-Density Generator-Matrix (LDGM) codes for (lossy) source coding [12]) in conjunction with iterative message-passing algorithms (e.g., the Sum-Product (SP) algorithm for channel decoding [11] and the BiasPropagation (BiP) algorithm for (lossy) source encoding [13], [14]). These basic coding components can serve as the building blocks of more sophisticated schemes for the problems at the second level of the hierarchy. Notable examples include the Gelfand-Pinsker problem and the Wyner-Ziv problem, which are solved via proper combination of source codes and channel codes [15], [16]. With these solutions in hand, one can then tackle the problems at the third level or even higher. From this perspective, our proposed scheme for the CEO problem can be interpreted as successive implementation of Wyner-Ziv coding.

The conversion of the CEO problem to the WynerZiv problem is realized using quantization splitting. The idea of quantization splitting is by no means new. Indeed, it has been applied to the multiterminal source coding problem [17] and multiple description problem [18] among others [19], particularly in the quadratic Gaussian setting. However, to the best of our knowledge, the application of quantization splitting is mainly restricted in the theoretical domain as a conceptual apparatus, and its practical implementation has not been addressed in the literature, at least for the problem under consideration (namely, the CEO problem under log-loss). In this work we mainly focus on the setting where the source is binary-symmetric and is corrupted by independent Bernoulli noises. It is worth emphasizing that this simple setting captures the essential features of the CEO problem and the methodology underlying our proposed scheme is in fact broadly applicable.

The organization of this paper is as follows. The problem definition and the concept of quantization splitting are presented in Section II. The proposed scheme is described in Section III. Sections IV and V contain associated analytical and numerical results, respectively. We conclude the paper in Section VI.

\section{The CEO Problem and Quantization SPlitTing}

\section{A. Notations}

Throughout this paper, the logarithm is to the base 2. Random variables and their realizations are shown by capital letters and lowercase letters in italics, respectively. Sets and alphabet set of random variables are depicted by calligraphic letters. Furthermore, matrices are shown by bold-faced letters. The binary entropy function is $h_{b}(x)=-x \log x-(1-x) \log (1-x), \mathbb{B} \triangleq\{0,1\}$, and $p * d=p(1-d)+(1-p) d$ shows the binary convolution of $p$ and $d$. The list of some symbols used in the paper is represented in Table $\mathrm{I}$.

\section{B. System Model}

Let $X^{n}=\left(X_{1}, \cdots, X_{n}\right)$ be an independent and identically distributed (i.i.d.) remote source. $L$ noisy observations of

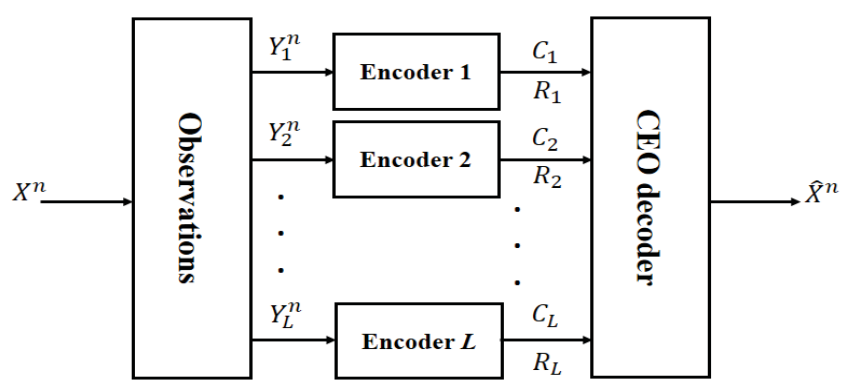

Fig. 1: Configuration of an $L$-link CEO problem.

$X^{n}$ are available in $L$ links that are mutually independent without any communication among them. These noisy observations, $Y_{l}^{n}$ for $l \in \mathcal{I}_{L} \triangleq\{1, \cdots, L\}$, are generated by $X^{n}$ through independent memoryless channels. The block diagram of an $L$-link CEO problem is depicted in Fig. 1. In each link, an encoder maps its noisy observation to a codeword $C_{l}$ by using a function $f_{l}$, as follows:

$$
C_{l}=f_{l}\left(Y_{l}^{n}\right) \text {, where } Y_{l}^{n} \in \mathcal{Y}_{l}^{n} \text { and } C_{l} \in C_{l} \text {, for } l \in \mathcal{I}_{L} \text {. }
$$

The codewords $C_{l}$, for $l \in \mathcal{I}_{L}$, are sent to a joint CEO decoder via noiseless channels. The CEO decoder produces a soft reconstruction $\hat{X}^{n}=\left(\hat{X}_{1}, \cdots, \hat{X}_{n}\right)$ of the original remote source $X^{n}$ by using a function $g$, as follows:

$$
\hat{X}^{n}=g\left(C_{1}, \cdots, C_{L}\right) \text {, where }\left(C_{1}, \cdots, C_{L}\right) \in C_{1} \cdots \times C_{L} .
$$

Definition 1: The log-loss induced by a symbol $x \in \mathcal{X}$ and a probability distribution $\hat{x}$ on $\mathcal{X}$ is defined as

$$
d(x, \hat{x})=\log \left(\frac{1}{\hat{x}(x)}\right) .
$$

More generally, for a sequence of symbols $x^{n}=\left(x_{1}, \cdots, x_{n}\right)$ and a sequence of distributions $\hat{x}^{n}=\left(\hat{x}_{1}, \cdots, \hat{x}_{n}\right)$, let

$$
d\left(x^{n}, \hat{x}^{n}\right)=\frac{1}{n} \sum_{t=1}^{n} \log \left(\frac{1}{\hat{x}_{t}\left(x_{t}\right)}\right) .
$$

Definition 2: A rate-distortion vector $\left(R_{1}, \cdots, R_{L}, D\right)$ is called strict-sense achievable under log-loss, if for all sufficiently large $n$, there exist functions $f_{1}, f_{2}, \ldots, f_{L}$, and $g$ respectively according to (1) and (2) such that

$$
\begin{aligned}
R_{l} & \geq \frac{1}{n} \log \left|C_{l}\right|, \text { for } l \in \mathcal{I}_{L} ; \\
D & \geq \mathbb{E}\left(d\left(X^{n}, \hat{X}^{n}\right)\right),
\end{aligned}
$$

where $\mathbb{E}(\cdot)$ denotes expectation function. The closure of the set of all strict-sense achievable vectors $\left(R_{1}, \cdots, R_{L}, D\right)$ is called the rate-distortion region of the CEO problem under log-loss and is denoted by $\overline{\mathcal{R D}}_{\mathrm{CEO}}^{\star}$.

Definition 3 ( [9, Definition 7]): Let $\mathcal{R} \mathcal{D}_{\mathrm{CEO}}^{i}$ be the set of all $\left(R_{1}, \cdots, R_{L}, D\right)$ satisfying

$$
\sum_{l \in \mathcal{A}} R_{l} \geq I\left(Y_{\mathcal{A}} ; U_{\mathcal{A}} \mid U_{\mathcal{A}^{c}}, Q\right), \quad \emptyset \subset \mathcal{A} \subseteq \mathcal{I}_{L},
$$

$$
D \geq H\left(X \mid U_{\mathcal{I}_{L}}, Q\right),
$$


TABLE I: LIST OF SOME SYMBOLS USED IN THIS PAPER.

\begin{tabular}{|c|c|}
\hline Symbol & Description \\
\hline$q_{j}$ & Appearance probability of the binary representation of $j$ in the links' output \\
\hline$Q_{j}$ & Appearance probability of the binary representation of $j$ in the links' output for a specific $x \in \mathbb{B}$ \\
\hline$p_{l}$ & Bernoulli noise parameter in the $l$-th link \\
\hline$Y_{l}^{n}$ & Binary noisy observation in the $l$-th link \\
\hline$d_{l}$ & Crossover probability of the binary symmetric test channel in the $l$-th link \\
\hline$P_{l}$ & Mamming distance between remote source and quantized sequence in the $l$-th link \\
\hline$\phi_{i}(\cdot)$ & Mapping from $\mathbb{B}^{K_{i}}$ to $\mathbb{B}$ \\
\hline$\mu_{\max }$ & Number of syndrome bits in compound LDGM-LDPC codes \\
\hline$k_{i}$ and $k_{i}^{\prime}$ & Number of variable nodes in LDGM code $C_{W_{l}}$ of the $l$-th link \\
\hline$m_{l}$ & Number of variable nodes in LDGM code $C_{i}^{\prime}$ of the $i$-th link \\
\hline$M_{i}$ & Observation noise in the $l$-th link \\
\hline$N_{l}^{n}$ & Output sequence of splitter in the $l$-th link \\
\hline$W_{l}$ & Quantized sequence in the $l$-th link \\
\hline$U_{l}$ & Remote binary symmetric source $(n$-tuple) \\
\hline$X^{n}$ & Slope of the tangent line to sum rate-distortion bound curve \\
\hline$\mu^{n}$ & Soft reconstruction of the remote source \\
\hline$\hat{X}^{n}$ & Splitting parameter in the $l$-th link \\
\hline$\delta_{l}$ & Theoretical bound of Sum-rate (Distortion) \\
\hline$R_{\mathrm{th}}\left(D_{\mathrm{th}}\right)$ & Well-ordered permutation \\
\hline$\sigma$ & \\
\hline
\end{tabular}

for some joint distribution

$$
p_{Q}(q) p_{X}(x) \prod_{l=1}^{L} p_{Y_{l} \mid X}\left(y_{l} \mid x\right) p_{U_{l} \mid Y_{l}, Q}\left(u_{l} \mid y_{l}, q\right),
$$

where in (6a), $Y_{\mathcal{A}}=\left\{Y_{l}: l \in \mathcal{A}\right\}$ and $\mathcal{A}^{c}=\mathcal{I}_{L} \backslash \mathcal{A}$.

Definition 4 ([9, Definition 8]): Let $\mathcal{R} \mathcal{D}_{\mathrm{CEO}}^{o}$ be the set of all $\left(R_{1}, \cdots, R_{L}, D\right)$ satisfying

$$
\sum_{l \in \mathcal{A}} R_{l} \geq\left[\sum_{l \in \mathcal{A}} I\left(Y_{l} ; U_{l} \mid X, Q\right)+H\left(X \mid U_{\mathcal{A}^{c}}, Q\right)-D\right]^{+}, \emptyset \subset \mathcal{A} \subseteq \mathcal{I}_{L},
$$

and (6b), for some joint distribution (7), where $[x]^{+}=$ $\max \{0, x\}$ and $U_{\mathcal{A}} \leftrightarrow Y_{\mathcal{A}} \leftrightarrow X \leftrightarrow Y_{\mathcal{A}^{c}} \leftrightarrow U_{\mathcal{A}^{c}}$ forms a Markov chain for any $\mathcal{A} \subseteq \mathcal{I}_{L}$.

It is shown in [9] that

$$
\overline{\mathcal{R D}}_{\mathrm{CEO}}^{\star}=\mathcal{R} \mathcal{D}_{\mathrm{CEO}}^{i}=\mathcal{R} \mathcal{D}_{\mathrm{CEO}}^{o} ;
$$

moreover, there is no loss of generality in imposing the cardinality bounds $\left|\mathcal{U}_{l}\right| \leq\left|\mathcal{Y}_{l}\right|, l \in \mathcal{I}_{L}$ and $|Q| \leq L+2$ on the alphabet sizes of auxiliary random variables $U_{l}$ and timesharing variable $Q$, respectively.

Given test channels $p_{U_{l} \mid Y_{l}}, \quad l \in \mathcal{I}_{L}$, we define $\mathcal{R} \mathcal{D}_{\mathrm{CEO}}\left(p_{U_{l} \mid Y_{l}}, l \in \mathcal{I}_{L}\right)$ as the set of all $\left(R_{1}, \cdots, R_{L}, D\right)$ satisfying

$$
\begin{aligned}
\sum_{l \in \mathcal{A}} R_{l} & \geq I\left(Y_{\mathcal{A}} ; U_{\mathcal{A}} \mid U_{\mathcal{A}^{c}}\right), \quad \emptyset \subset \mathcal{A} \subseteq \mathcal{I}_{L}, \\
D & \geq H\left(X \mid U_{\mathcal{I}_{L}}\right),
\end{aligned}
$$

where $X, Y_{I_{L}}$, and $U_{I_{L}}$ are jointly distributed according to $p_{X}(x) \prod_{l=1}^{L} p_{Y_{l} \mid X}\left(y_{l} \mid x\right) p_{U_{l} \mid Y_{l}}\left(u_{l} \mid y_{l}\right)$.

Note that (10) and (11) respectively correspond to (6a) and (6b) with timesharing variable $Q$ set to be a constant. Therefore, $\mathcal{R} \mathcal{D}_{\mathrm{CEO}}^{i}$ (as well as $\mathcal{R} \mathcal{D}_{\mathrm{CEO}}^{o}$ and $\overline{\mathcal{R D}}_{\mathrm{CEO}}^{\star}$ in light of (9)) can be expressed as the convex hull of the union of $\mathcal{R} \mathcal{D}_{\mathrm{CEO}}\left(p_{U_{l} \mid Y_{l}}, l \in \mathcal{I}_{L}\right)$ over all $\left(p_{U_{l} \mid Y_{l}}, l \in \mathcal{I}_{L}\right)$. Moreover, we define $\mathcal{R}_{\mathrm{CEO}}\left(p_{U_{l} \mid Y_{l}}, l \in \mathcal{I}_{L}\right)$ to be the set of all $\left(R_{1}, \cdots, R_{L}\right)$ satisfying (10) and define its dominant face, denoted by $\mathcal{F}_{\mathrm{CEO}}\left(p_{U_{l} \mid Y_{l}}, l \in \mathcal{I}_{L}\right)$, to be the set of $\left(R_{1}, \cdots, R_{L}\right) \in \mathcal{R}_{\mathrm{CEO}}\left(p_{U_{l} \mid Y_{l}}, l \in \mathcal{I}_{L}\right)$ satisfying $\sum_{l=1}^{L} R_{l}=I\left(Y_{I_{L}} ; U_{I_{L}}\right)$. Due to the contrapolymatroid structure of $\mathcal{R}_{\mathrm{CEO}}\left(p_{U_{l} \mid Y_{l}}, l \in \mathcal{I}_{L}\right)$ [17], $[19], \quad \mathcal{F}_{\mathrm{CEO}}\left(p_{U_{l} \mid Y_{l}}, l \in \mathcal{I}_{L}\right)$ is non-empty and every $\left(R_{1}, \cdots, R_{L}, D\right)$ in $\mathcal{R} \mathcal{D}_{\mathrm{CEO}}\left(p_{U_{l} \mid Y_{l}}, l \in \mathcal{I}_{L}\right)$ is dominated, in a component-wise manner, by $\left(R_{1}^{\prime}, \cdots, R_{L}^{\prime}, H\left(X \mid U_{I_{L}}\right)\right)$ for some $\left(R_{1}^{\prime}, \cdots, R_{L}^{\prime}\right) \in \mathcal{F}_{\mathrm{CEO}}\left(p_{U_{l} \mid Y_{l}}, l \in \mathcal{I}_{L}\right)$.

\section{Quantization Splitting}

$\mathcal{F}_{\mathrm{CEO}}\left(p_{U_{l} \mid Y_{l}}, l \in \mathcal{I}_{L}\right)$ has $L$ ! corner points. Specifically, each permutation $\pi$ on $\mathcal{I}_{L}$ is associated with a corner point $\left(R_{1}(\pi), \cdots, R_{L}(\pi)\right)$ of $\mathcal{F}_{\mathrm{CEO}}\left(p_{U_{l} \mid Y_{l}}, l \in \mathcal{I}_{L}\right)$ as follows:

$$
\begin{aligned}
R_{\pi(l)}(\pi) & =I\left(Y_{\pi(l)} ; U_{\pi(l)} \mid U_{\pi(l+1)}, \cdots, U_{\pi(L)}\right), \quad l \in \mathcal{I}_{L-1}, \\
R_{\pi(L)}(\pi) & =I\left(Y_{\pi(L)} ; U_{\pi(L)}\right) .
\end{aligned}
$$

These corner points can be achieved via successive WynerZiv coding with decoding order $U_{\pi(L)} \rightarrow U_{\pi(L-1)} \rightarrow \cdots \rightarrow$ $U_{\pi(1)}$ (an implementation of this scheme for the case $L=2$ can be found in [20]).

To achieve non-corner points of $\mathcal{F}_{\mathrm{CEO}}\left(p_{U_{l} \mid Y_{l}}, l \in \mathcal{I}_{L}\right)$, we employ the quantization splitting technique introduced in [17], which is a generalization of the source splitting technique [21] and a counterpart of the rate splitting technique in channel coding [22], [23]. Roughly speaking, the basic idea underlying the quantization splitting technique is that each non-corner point in the $L$-dimensional space can be projected to a corner point in the $(2 L-1)$-dimensional space. Specifically, it is known [17, Theorem 2.1] that, for any rate tuple $\left(R_{1}, \cdots, R_{L}\right) \in \mathcal{F}_{\mathrm{CEO}}\left(p_{U_{l} \mid Y_{l}}, l \in \mathcal{I}_{L}\right)$, there exist random variables $W_{l}, l \in \mathcal{I}_{L}$, and a well-ordered permutation $\sigma^{1}$ on the set $\left\{W_{1}, \cdots, W_{L}, U_{1}, \cdots, U_{L}\right\}$ such that

$$
R_{l}=I\left(Y_{l} ; W_{l} \mid\left\{W_{l}\right\}_{\sigma}^{-}\right)+I\left(Y_{l} ; U_{l} \mid\left\{U_{l}\right\}_{\sigma}^{-}\right), \quad l \in \mathcal{I}_{L},
$$

${ }^{1} \mathrm{~A}$ well-ordered permutation is an arbitrary ordering of the set $\left\{W_{1}, \cdots, W_{L}, U_{1}, \cdots, U_{L}\right\}$ with $W_{l}$ appearing before $U_{l}$ for all $l \in$ $\mathcal{I}_{L}$. 
where $\left\{W_{l}\right\}_{\sigma}^{-}$and $\left\{U_{l}\right\}_{\sigma}^{-}$represent the set of random variables that respectively appear before $W_{l}$ and $U_{l}$ in the well-ordered permutation $\sigma$; moreover, $W_{l}$ is a physically degraded version $U_{l}, l \in \mathcal{I}_{L}$, and at least one $W_{l}$ is independent of $U_{l}$ (and thus can be eliminated).

It is instructive to view $U_{l}$ as a fine-description of $Y_{l}$ and view $W_{l}$ as a coarse-description split from $U_{l}, l \in \mathcal{I}_{L}$. Eq. (12) suggests that the given rate tuple $\left(R_{1}, \cdots, R_{L}\right)$ can be achieved via successive Wyner-Ziv coding with decoding order specified by $\sigma$. It should be emphasized that the successive Wyner-Ziv coding scheme for non-corner points is in general more complicated than that for corner points. First of all, the scheme for non-corner points involves more encoding and decoding steps. Secondly and more importantly, to realize the splitting effect, one needs to generate a coarse-description codebook and then, for each of its codewords, generate a fine-description codebook; as a consequence, the number of fine-description codebooks grows exponentially with the codeword length, causing a serious problem in practice. In this work we circumvent this problem by using a codebook construction technique inspired by the functional representation lemma [24], [25]. Successive refinement coding scheme is also a multiterminal encoding problem for, basically, downlink, where terminals are classified into several groups, each having different distortion requirements. The remote source is encoded such that the description for the groups having higher distortion requirement can help recover another groups having lower distortion requirement. Alternatively, our proposed coding scheme successively decodes binary observations and then softly reconstructs the remote source with a single value of distortion under the log-loss criterion.

\section{Description of the Proposed Scheme}

Consider an $L$-link binary CEO problem, where a remote Binary-Symmetric Source (BSS) is corrupted by independent Bernoulli noises with parameters $p_{1}, p_{2}, \ldots$ , and $p_{L}$, i.e.,

$$
X \sim \operatorname{Ber}\left(\frac{1}{2}\right), \quad Y_{l}=X \oplus N_{l}, \quad N_{l} \sim \operatorname{Ber}\left(p_{l}\right), \quad l \in \mathcal{I}_{L} .
$$

We make the following two assumptions.

1) A binary-symmetric test channel model is adopted for each encoder. More specifically, it is assumed that $p_{U_{l} \mid Y_{l}}$ is a Binary-Symmetric Channel (BSC) with crossover probability $d_{l}, l \in \mathcal{I}_{L}$. Hence, we can write $U_{l}=Y_{l} \oplus Z_{l}, l \in \mathcal{I}_{L}$, where $Z_{l} \sim \operatorname{Ber}\left(d_{l}\right), l \in \mathcal{I}_{L}$, are mutually independent and are independent of $\left(X, Y_{I_{L}}\right)$ as well. This assumption is justified by the numerical results in [20].

2) A BSC model is adopted for each splitter. More specifically, it is assumed that $p_{W_{l} \mid U_{l}}$ is a BSC with crossover probability $\delta_{l}, l \in \mathcal{I}_{L}$. Hence, we can write $W_{l}=U_{l} \oplus V_{l}, l \in \mathcal{I}_{L}$, where $V_{l} \sim \operatorname{Ber}\left(\delta_{l}\right), l \in \mathcal{I}_{L}$, are mutually independent and are independent of $\left(X, Y_{\mathcal{I}_{L}}, U_{\mathcal{I}_{L}}\right)$ as well. According to [23, Definition 2], this assumption incurs no loss of generality.
Since the coding schemes associated with different wellordered permutations are conceptually similar, for ease of exposition, we focus on a specific permutation $\sigma=$ $\left(W_{1}, \cdots, W_{L-1}, U_{L}, U_{L-1}, \cdots, U_{1}\right)$ (we eliminate $W_{L}$ by setting $\delta_{L}=\frac{1}{2}$ ). Each conditional mutual information in (12) can be written as the difference of two terms, one associated with quantization and the other with binning. As an example, consider the second term of $R_{1}$, i.e., $I\left(Y_{1} ; U_{1} \mid W_{1}, \cdots, W_{L-1}, U_{2}, \cdots, U_{L}\right)$. We have

$$
\begin{aligned}
& I\left(Y_{1} ; U_{1} \mid W_{1}, \cdots, W_{L-1}, U_{2}, \cdots, U_{L}\right) \\
& =I\left(Y_{1} ; U_{1} \mid W_{1}, U_{2}, \cdots, U_{L}\right) \\
& =I\left(U_{2}, \cdots, U_{L}, Y_{1} ; U_{1} \mid W_{1}\right)-I\left(U_{2}, \cdots, U_{L} ; U_{1} \mid W_{1}\right) \\
& =I\left(Y_{1} ; U_{1} \mid W_{1}\right)-I\left(U_{2}, \cdots, U_{L} ; U_{1} \mid W_{1}\right),
\end{aligned}
$$

where (14) is due to the degradeness of $W_{l}$ with respect to $U_{l}, l \in[2: L-1]$, where $[j: k] \triangleq\{j, \cdots, k\}$, and $(15)$ is because of the fact that $\left(U_{1}, W_{1}\right)$ and $\left(U_{2}, \cdots, U_{L}\right)$ are conditionally independent given $Y_{1}$. The term $I\left(Y_{1} ; U_{1} \mid W_{1}\right)$ specifies the quantization rate needed to generate the finedescription $U_{1}$ given the coarse description $W_{1}$ while the term $I\left(U_{2}, \cdots, U_{L} ; U_{1} \mid W_{1}\right)$ specifies the amount of rate reduction achievable through binning.

We use a binary quantizer to map the outputs of a BSS to the codewords of an LDGM code with the minimum Hamming distance. These quantizers are utilized in the encoders of our proposed coding scheme. Practically, binary quantization can be realized by using some iterative message passing algorithms such as the BiP algorithm [13] or the survey-propagation algorithm [12]. Presence of side information can further reduce the compression rate required for a prescribed distortion constraint. Actually, this lossless source coding scenario can be practically realized by a binning operation based on channel coding schemes [4]. In our proposed coding scheme, binning is implemented by using LDPC codes with the syndrome generation scheme. This binning scheme is also used for the asymmetric Slepian-Wolf coding problem. In practice, the SP algorithm can be used to iteratively decode the LDPC coset code specified by the given syndrome.

\section{A. The Proposed Coding Scheme: an Information- Theoretic Description}

To elucidate the overall structure of the proposed scheme, we first give a short description using the information-theoretic terminology. First, let $W_{L} \triangleq U_{L}$. In the following description, all the $\epsilon$ quantities are small positive real numbers.

\section{Codebook Generation:}

1) For $l \in \mathcal{I}_{L}$, a codebook $C_{W_{l}}$ of rate $I\left(Y_{l} ; W_{l}\right)+\epsilon_{l, 1}$ should be constructed with each codeword generated independently according to $\prod_{t=1}^{n} p_{W_{l}}(\cdot)$.

2) For $i \in \mathcal{I}_{L-1}$ and each codeword $w_{i}^{n} \in C_{W_{i}}$, a codebook $C_{U_{i}}\left(w_{i}^{n}\right)$ of rate $I\left(Y_{i} ; U_{i} \mid W_{i}\right)+\epsilon_{i, 2}$ is required with each codeword generated independently according to $\prod_{t=1}^{n} p_{U_{i} \mid W_{i}}\left(\cdot \mid w_{i, t}\right)$. 
3) For $i \in[2: L]$, the $C_{W_{i}}$ should be partitioned into $2^{n\left[I\left(Y_{i} ; W_{i} \mid W_{1}, \cdots, W_{i-1}\right)+\epsilon_{i, 3}\right]}$ bins, where each bin contains $2^{n\left[I\left(W_{1}, \cdots, W_{i-1} ; W_{i}\right)-\epsilon_{i, 3}\right]}$ codewords.

4) For $i \in I_{L-1}$ and each codeword $w_{i}^{n} \in$ $C_{W_{i}}, \quad C_{U_{i}}\left(w_{i}^{n}\right)$ should be partitioned into $2^{n\left[I\left(Y_{i} ; U_{i} \mid W_{1}, \cdots, W_{i}, U_{i+1}, \cdots, U_{L}\right)+\epsilon_{i, 4}\right]}$ bins, where each bin contains $2^{n\left[I\left(W_{1}, \cdots, W_{i-1}, U_{i+1}, \cdots, U_{L} ; U_{i} \mid W_{i}\right)-\epsilon_{i, 4}\right]}$ codewords.

\section{Encoding:}

1) For $l \in \mathcal{I}_{L}$ and a given $y_{l}^{n}$, the $l$-th encoder finds a codeword $w_{l}^{n} \in \mathcal{C}_{W_{l}}$ that is jointly typical with $y_{l}^{n}$. Note that the Hamming distance between $w_{l}^{n}$ and $y_{l}^{n}$ is approximately $n\left(d_{l} * \delta_{l}\right)$.

2) For $i \in \mathcal{I}_{L-1}$, the $i$-th encoder finds a codeword $u_{i}^{n} \in C_{U_{i}}\left(w_{i}^{n}\right)$ that is jointly typical with $\left(y_{i}^{n}, w_{i}^{n}\right)$. Note that the Hamming distance between $u_{i}^{n}$ and $y_{i}^{n}$ is approximately $n d_{i}$ while the Hamming distance between $u_{i}^{n}$ and $w_{i}^{n}$ is approximately $n \delta_{i}$.

3) For $l \in \mathcal{I}_{L}$, the $l$-th encoder sends the index $b\left(w_{l}^{n}\right)$ of the bin that contains $w_{l}^{n}$ (for $l=1$, it only sends the index $i\left(w_{1}^{n}\right)$ of $w_{1}^{n}$, and for $l=L$ nothing is sent), and the index $b\left(u_{l}^{n}\right)$ of the bin that contains $u_{l}^{n}$ to the decoder.

\section{Decoding:}

1) The decoder first decodes $w_{1}^{n}$ based on $i\left(w_{1}^{n}\right)$.

2) For $i \in[2: L]$, it decodes $w_{i}^{n}$ by searching in the bin with index $b\left(w_{i}^{n}\right)$ for the unique codeword that is jointly typical with $\left(w_{1}^{n}, w_{2}^{n}, \cdots, w_{i-1}^{n}\right)$.

3) For $j \in[L-1: 1]$, it decodes $u_{j}^{n}$ by searching in the bin with index $b\left(u_{j}^{n}\right)$ for the unique codeword that is jointly typical with $\left(w_{1}^{n}, \cdots, w_{j}^{n}, u_{j+1}^{n}, \cdots, u_{L}^{n}\right)$.

4) Finally, it uses $\left(\hat{u}_{1}^{n}, \cdots, \hat{u}_{L}^{n}\right)$ to produce a soft reconstruction of $x^{n}$ by the following rule:

$$
\hat{x}_{t}=p_{X \mid U_{I_{L}}}\left(\cdot \mid \hat{u}_{1, t}, \cdots, \hat{u}_{L, t}\right), \quad t \in \mathcal{I}_{n} .
$$

The

$$
\text { conditional probability }
$$

function

$p_{X \mid U_{I_{L}}}\left(\cdot \mid \hat{u}_{1, t}, \cdots, \hat{u}_{L, t}\right)$ depends on the binary values of $\hat{u}_{l, t}$, for $l \in \mathcal{I}_{L}$. This function can be determined based on the joint distribution diagram of the CEO problem. As an example for $L=3$, we calculate $p_{X \mid U_{1}, U_{2}, U_{3}}\left(x_{j} \mid \hat{u}_{1, j}, \hat{u}_{2, j}, \hat{u}_{3, j}\right)$ for $\left(x_{j}=0, \hat{u}_{1, j}=1, \hat{u}_{2, j}=0, \hat{u}_{3, j}=1\right)$. The other 15 cases can be calculated similarly.

$$
\begin{aligned}
& \hat{x}_{j}=p_{X \mid U_{1}, U_{2}, U_{3}}(0 \mid 1,0,1)=\frac{p_{X, U_{1}, U_{2}, U_{3}}(0,1,0,1)}{p_{U_{1}, U_{2}, U_{3}}(1,0,1)} \\
& =\frac{p_{U_{1}, U_{2}, U_{3} \mid X}(1,0,1 \mid 0) \times 0.5}{p_{U_{1}, U_{2}, U_{3} \mid X}(1,0,1 \mid 0) \times 0.5+p_{U_{1}, U_{2}, U_{3} \mid X}(1,0,1 \mid 1) \times 0.5} \\
& =\frac{\tau_{1}}{\tau_{1}+\tau_{2}},
\end{aligned}
$$

where $\tau_{1}=\left(p_{1} * d_{1}\right)\left(1-p_{2} * d_{2}\right)\left(p_{3} * d_{3}\right)$ and $\tau_{2}=\left(1-p_{1} *\right.$ $\left.d_{1}\right)\left(p_{2} * d_{2}\right)\left(1-p_{3} * d_{3}\right)$.

\section{B. The Proposed Coding Scheme: a Coding-Theoretic De- scription}

Now we translate the above information-theoretic description of the proposed scheme to a coding-theoretic description. Along the way, we address certain practical issues encountered in codebook generation using a construction technique inspired by the functional representation lemma. For notational simplicity, the description is given for the case $L=3$; the extension to the general case is straightforward.

\section{Codebook Generation:}

1) For $l \in \mathcal{I}_{3}$, an LDGM codebook $C_{W_{l}}$ should be generated with the rate of $I\left(Y_{l} ; W_{l}\right)+\epsilon_{l, 1}=1-h_{b}\left(d_{l} * \delta_{l}\right)+\epsilon_{l, 1}$ 2 .

2) For $i \in \mathcal{I}_{2}$ and each codeword $w_{i}^{n}$, a codebook $C_{U_{i}}\left(w_{i}^{n}\right)$ is constructed as follows ${ }^{3}$ :

Firstly, an LDGM code $C_{i}^{\prime}$ should be considered with $2^{n\left[I\left(Y_{i} ; U_{i} \mid W_{i}\right)+\epsilon_{i, 2}\right]}$ codewords with each of length $n K_{i}$, where $K_{i}$ is a fixed integer. Let $\phi_{i}(\cdot)$ be a mapping ${ }^{4}$ from $\mathbb{B}^{K_{i}} \rightarrow \mathbb{B}$ such that

$$
\left|S_{0}\right| \approx 2^{K_{i}}\left(1-\delta_{i}\right) \text { and }\left|S_{1}\right| \approx 2^{K_{i}} \delta_{i},
$$

where $S_{b} \triangleq\left\{s^{K_{i}} \in \mathbb{B}^{K_{i}}: \phi_{i}\left(s^{K_{i}}\right)=b\right\}, b \in \mathbb{B}$. Note that the approximation in (18) can be made arbitrarily precise when $K_{i} \rightarrow \infty$. For each codeword $c^{n K_{i}} \triangleq\left(c_{1}, \cdots, c_{n K_{i}}\right) \in C_{i}^{\prime}$, the $c^{n K_{i}}$ is mapped to a codeword of length $n$ by using $\phi_{i}(\cdot)$ as below:

$$
\left(\phi_{i}\left(c_{1}, \cdots, c_{K_{i}}\right), \cdots, \phi_{i}\left(c_{(n-1) K_{i}+1}, \cdots, c_{n K_{i}}\right)\right) .
$$

By doing this for all codewords in $C_{i}^{\prime}$, a new codebook $\phi_{i}\left(C_{i}^{\prime}\right)$ is obtained with $2^{n\left[I\left(Y_{i} ; U_{i} \mid W_{i}\right)+\epsilon_{i, 2}\right]}$ codewords, each of length $n$. Hence, the codebook $C_{U_{i}}\left(w_{i}^{n}\right)$ can be defined as $w_{i}^{n} \oplus \phi_{i}\left(C_{i}^{\prime}\right)$, which is a codebook obtained by adding $w_{i}^{n}$ to each codeword in $\phi_{i}\left(C_{i}^{\prime}\right)$. Now consider the backward channels $Y_{i}=U_{i} \oplus Z_{i}^{\prime}$ and $U_{i}=W_{i} \oplus V_{i}^{\prime}{ }^{5}$, where $Z_{i}^{\prime} \sim \operatorname{Ber}\left(d_{i}\right), V_{i}^{\prime} \sim \operatorname{Ber}\left(\delta_{i}\right)$, and $W_{i}$ are mutually independent. It can be verified that

$$
I\left(Y_{i} ; U_{i} \mid W_{i}\right)=I\left(V_{i}^{\prime} \oplus Z_{i}^{\prime} ; V_{i}^{\prime}\right)=h_{b}\left(\delta_{i} * d_{i}\right)-h_{b}\left(d_{i}\right) .
$$

3) For $i=2,3$, to partition $C_{W_{i}}$ into $2^{n\left[I\left(Y_{i} ; W_{i} \mid W_{1}, \cdots, W_{i-1}\right)+\epsilon_{i, 3}\right]}$ bins with each bin containing $2^{n\left[I\left(W_{1}, \cdots, W_{i-1} ; W_{i}\right)-\epsilon_{i, 3}\right]}$ codewords, an LDPC code of rate $I\left(W_{1}, \cdots, W_{i-1} ; W_{i}\right)-\epsilon_{i, 3}$ is used with parity-check matrix $\mathbf{H}_{i}=\left(\tilde{\mathbf{H}}_{i}, \hat{\mathbf{H}}_{i}\right)$, where $\tilde{\mathbf{H}}_{i}$ is the parity-check matrix of $C_{W_{i}}$. It can be verified that

$$
\begin{aligned}
& I\left(Y_{2} ; W_{2} \mid W_{1}\right)= \\
& I\left(V_{1}^{\prime} \oplus Z_{1}^{\prime} \oplus N_{1}^{\prime} \oplus N_{2} ; V_{1}^{\prime} \oplus Z_{1}^{\prime} \oplus N_{1}^{\prime} \oplus N_{2} \oplus Z_{2} \oplus V_{2}\right) \\
& =H\left(V_{1}^{\prime} \oplus Z_{1}^{\prime} \oplus N_{1}^{\prime} \oplus N_{2} \oplus Z_{2} \oplus V_{2}\right)-H\left(Z_{2} \oplus V_{2}\right) \\
& =h_{b}\left(\delta_{1} * d_{1} * p_{1} * p_{2} * d_{2} * \delta_{2}\right)-h_{b}\left(d_{2} * \delta_{2}\right), \\
& I\left(W_{1} ; W_{2}\right)=1-H\left(V_{1}^{\prime} \oplus Z_{1}^{\prime} \oplus N_{1}^{\prime} \oplus N_{2} \oplus Z_{2} \oplus V_{2}\right)
\end{aligned}
$$

\footnotetext{
${ }^{2}$ Note that $\delta_{3}=0$.

${ }^{3}$ This construction is inspired by the functional representation lemma.

${ }^{4}$ This is known as Gallager's mapping [26], which is widely used to construct source or channel codes with non-uniform empirical distribution [27], [28].

${ }^{5}$ The representation of such backward channels can be viewed as a manifestation of the functional representation lemma. Moreover, it is instructive to view $\phi_{i}\left(C_{i}^{\prime}\right)$ as a codebook generated by $V_{i}^{\prime}$.
} 


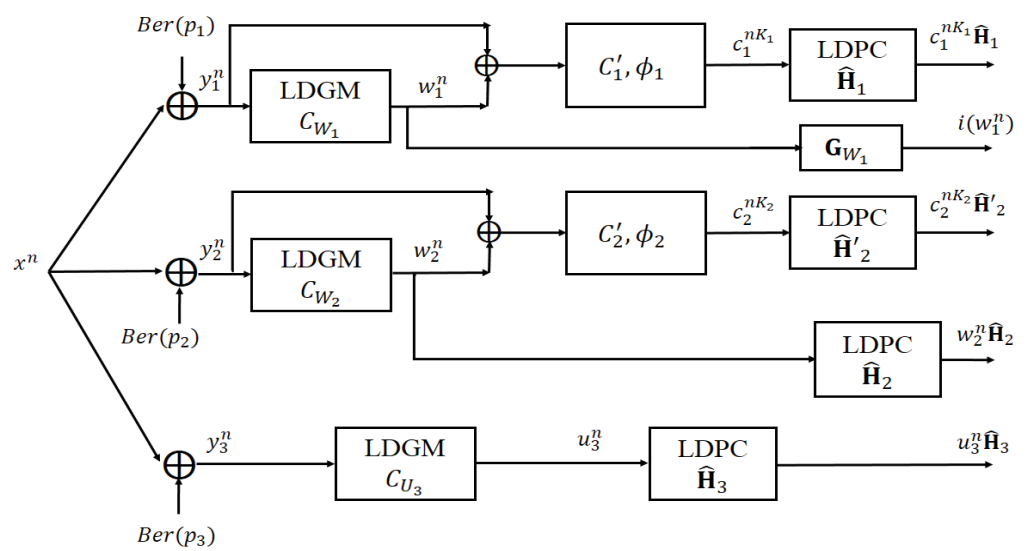

Fig. 2: The proposed encoding scheme.

$$
\begin{aligned}
& =1-h_{b}\left(\delta_{1} * d_{1} * p_{1} * p_{2} * d_{2} * \delta_{2}\right), \\
& I\left(W_{1}, W_{2} ; U_{3}\right)=H\left(W_{1}, W_{2}\right)-H\left(W_{1}, W_{2} \mid U_{3}\right) \\
& =1+h_{b}\left(\delta_{1} * d_{1} * p_{1} * p_{2} * d_{2} * \delta_{2}\right) \\
& -H\left(Z_{3}^{\prime} \oplus N_{3}^{\prime} \oplus N_{1} \oplus Z_{1} \oplus V_{1}, Z_{3}^{\prime} \oplus N_{3}^{\prime} \oplus N_{2} \oplus Z_{2} \oplus V_{2}\right) \\
& =H\left(W_{1}, W_{2}\right)-H\left(W_{1}, W_{2}, U_{3}\right)+1, \\
& I\left(Y_{3} ; U_{3} \mid W_{1}, W_{2}\right)=I\left(Y_{3} ; U_{3}\right)-I\left(W_{1}, W_{2} ; U_{3}\right) \\
& =1-h_{b}\left(d_{3}\right)-I\left(W_{1}, W_{2} ; U_{3}\right) .
\end{aligned}
$$

4) For $i \in \mathcal{I}_{2}$, to partition $C_{i}^{\prime}$ into $2^{n\left[I\left(Y_{i} ; U_{i} \mid W_{1}, \cdots, W_{i}, U_{i+1}, \cdots, U_{3}\right)+\epsilon_{i, 4}\right]}$ bins 6 with each bin containing $2^{n\left[I\left(W_{1}, \cdots, W_{i-1}, U_{i+1}, \cdots, U_{3} ; U_{i} \mid W_{i}\right)-\epsilon_{i, 4}\right]}$ codewords, an LDPC code is used with $2^{n\left(I\left(W_{1}, \cdots, W_{i-1}, U_{i+1}, \cdots, U_{3} ; U_{i} \mid W_{i}\right)-\epsilon_{i, 4}\right)}$ codewords, each of length $n K_{i}$. The parity-check matrix of this LDPC code is $\mathbf{H}_{1}=\left(\tilde{\mathbf{H}}_{1}, \hat{\mathbf{H}}_{1}\right)$ for $i=1$ and $\mathbf{H}_{2}^{\prime}=\left(\tilde{\mathbf{H}}_{2}^{\prime}, \hat{\mathbf{H}}_{2}^{\prime}\right)$ for $i=2$, where $\tilde{\mathbf{H}}_{1}$ and $\tilde{\mathbf{H}}_{2}^{\prime}$ are the parity-check matrices of $C_{1}^{\prime}$ and $C_{2}^{\prime}$, respectively. Thus, we have

$$
\begin{aligned}
& I\left(U_{2}, U_{3} ; U_{1} \mid W_{1}\right)= \\
& I\left(V_{1}^{\prime} \oplus Z_{1}^{\prime} \oplus N_{1}^{\prime} \oplus N_{2} \oplus Z_{2}, V_{1}^{\prime} \oplus Z_{1}^{\prime} \oplus N_{1}^{\prime} \oplus N_{3} \oplus Z_{3} ; V_{1}^{\prime}\right) \\
& =H\left(V_{1}^{\prime} \oplus Z_{1}^{\prime} \oplus N_{1}^{\prime} \oplus N_{2} \oplus Z_{2}, V_{1}^{\prime} \oplus Z_{1}^{\prime} \oplus N_{1}^{\prime} \oplus N_{3} \oplus Z_{3}\right) \\
& -H\left(Z_{1}^{\prime} \oplus N_{1}^{\prime} \oplus N_{2} \oplus Z_{2}, Z_{1}^{\prime} \oplus N_{1}^{\prime} \oplus N_{3} \oplus Z_{3}\right), \\
& I\left(Y_{1} ; U_{1} \mid W_{1}, U_{2}, U_{3}\right)=I\left(Y_{1} ; U_{1} \mid W_{1}\right)-I\left(U_{2}, U_{3} ; U_{1} \mid W_{1}\right), \\
& I\left(W_{1}, U_{3} ; U_{2} \mid W_{2}\right)= \\
& I\left(V_{2}^{\prime} \oplus Z_{2}^{\prime} \oplus N_{2}^{\prime} \oplus N_{1} \oplus Z_{1} \oplus V_{1}, V_{2}^{\prime} \oplus Z_{2}^{\prime} \oplus N_{2}^{\prime} \oplus N_{3} \oplus Z_{3} ; V_{2}^{\prime}\right) \\
& =H\left(V_{2}^{\prime} \oplus Z_{2}^{\prime} \oplus N_{2}^{\prime} \oplus N_{1} \oplus Z_{1} \oplus V_{1}, V_{2}^{\prime} \oplus Z_{2}^{\prime} \oplus N_{2}^{\prime} \oplus N_{3} \oplus Z_{3}\right) \\
& -H\left(Z_{2}^{\prime} \oplus N_{2}^{\prime} \oplus N_{1} \oplus Z_{1} \oplus V_{1}, Z_{2}^{\prime} \oplus N_{2}^{\prime} \oplus N_{3} \oplus Z_{3}\right), \\
& I\left(Y_{2} ; U_{2} \mid W_{1}, W_{2}, U_{3}\right)=I\left(Y_{2} ; U_{2} \mid W_{2}\right)-I\left(W_{1}, U_{3} ; U_{2} \mid W_{2}\right) .
\end{aligned}
$$

Encoding: Different from the information-theoretic description in Section III-A, we shall interpret joint typicality encoding as the minimum Hamming distance encoding, which is then implemented using the $\mathrm{BiP}$ algorithm.

1) For $l \in \mathcal{I}_{3}$ and a given $y_{l}^{n}$, the $l$-th encoder finds a codeword $w_{l}^{n} \in C_{W_{l}}$ from an LDGM code that is the closest (in the Hamming distance) to $y_{l}^{n}$.

${ }^{6}$ This induces a partition of $C_{U_{i}}\left(w_{i}^{n}\right)$.
2) For $i \in \mathcal{I}_{2}$, find a codeword $c_{i}^{n K_{i}} \in C_{i}^{\prime}$ such that $\phi_{i}\left(c_{i}^{n K_{i}}\right)$ is the closest (in the Hamming distance) to $y_{i}^{n} \oplus w_{i}^{n}$.

3) Send the index of $w_{1}^{n}$ and the syndrome $c_{1}^{n K_{1}} \hat{\mathbf{H}}_{1}$ from the first link to the decoder; note that $w_{1}^{n}=$ $i\left(w_{1}^{n}\right) \mathbf{G}_{W_{1}}$, where $\mathbf{G}_{W_{1}}$ is the generator matrix of LDGM code $C_{W_{1}}$. Also, send the syndromes $w_{2}^{n} \hat{\mathbf{H}}_{2}$ and $c_{2}^{n K_{2}} \hat{\mathbf{H}}_{2}^{\prime}$ from the second link to the decoder. Finally, send the syndrome $u_{3}^{n} \hat{\mathbf{H}}_{3}$ from the third link to the decoder.

The block diagram of the proposed encoding scheme is depicted in Fig. 2.

Decoding: Different from the information-theoretic description in Section III-A, we shall interpret joint typicality decoding as maximum a posteriori decoding, which is then implemented using the SP algorithm.

1) The decoder first sets $\hat{w}_{1}^{n}=w_{1}^{n}$.

2) It then finds the most likely choice of $w_{2}^{n}$, denoted by $\hat{w}_{2}^{n}$, based on $\hat{w}_{1}^{n}$ and $w_{2}^{n} \mathbf{H}_{2}$ (which can be deduced from $w_{2}^{n} \hat{\mathbf{H}}_{2}$ and the fact that $w_{2}^{n} \tilde{\mathbf{H}}_{2}$ is a zero vector). This can be realized via conventional Slepian-Wolf decoding with $\mathbf{H}_{2}$ defining the factor graph and $\hat{w}_{1}^{n}$ serving as side information (see, e.g., [29]).

3 ) It then finds the most likely choice of $u_{3}^{n}$, denoted by $\hat{u}_{3}^{n}$, based on $\hat{w}_{1}^{n}, \hat{w}_{2}^{n}$, and $u_{3}^{n} \mathbf{H}_{3}$ (which can be deduced from $u_{3}^{n} \hat{\mathbf{H}}_{3}$ and the fact that $u_{2}^{n} \tilde{\mathbf{H}}_{3}$ is a zero vector). This can be realized via conventional Slepian-Wolf decoding with $\mathbf{H}_{3}$ defining the factor graph and $\left(\hat{w}_{1}^{n}, \hat{w}_{2}^{n}\right)$ serving as side information.

4) It then finds the most likely choice of $c_{2}^{n K_{2}}$, denoted by $\hat{c}_{2}^{n K_{2}}$, based on $\hat{w}_{1}^{n}, \hat{w}_{2}^{n}, \hat{u}_{3}^{n}$, and $c_{2}^{n K_{2}} \mathbf{H}_{2}^{\prime}$ (which can be deduced from $c_{2}^{n K_{2}} \hat{\mathbf{H}}_{2}^{\prime}$ and the fact that $c_{2}^{n K_{2}} \tilde{\mathbf{H}}_{2}^{\prime}$ is a zero vector). This can be realized via joint demapping and decoding with $\left(\mathbf{H}_{2}^{\prime}, \phi_{2}\right)$ defining the factor graph and $\left(\hat{w}_{1}^{n}, \hat{w}_{2}^{n}, \hat{u}_{3}^{n}\right)$ serving as the channel output (see, e.g., [30]). Set $\hat{u}_{2}^{n}=\hat{w}_{2}^{n} \oplus \phi_{2}\left(\hat{c}_{2}^{n K_{2}}\right)$.

5) It then finds the most likely $c_{1}^{n K_{1}}$, denoted by $\hat{c}_{1}^{n K_{1}}$, based on $\hat{w}_{1}^{n}, \hat{u}_{2}^{n}, \hat{u}_{3}^{n}$, and $c_{1}^{n K_{1}} \mathbf{H}_{1}$ (which can be deduced from $c_{1}^{n K_{1}} \hat{\mathbf{H}}_{1}$ and the fact that $c_{1}^{n K_{1}} \tilde{\mathbf{H}}_{1}$ is a zero vector). This can be realized via joint 


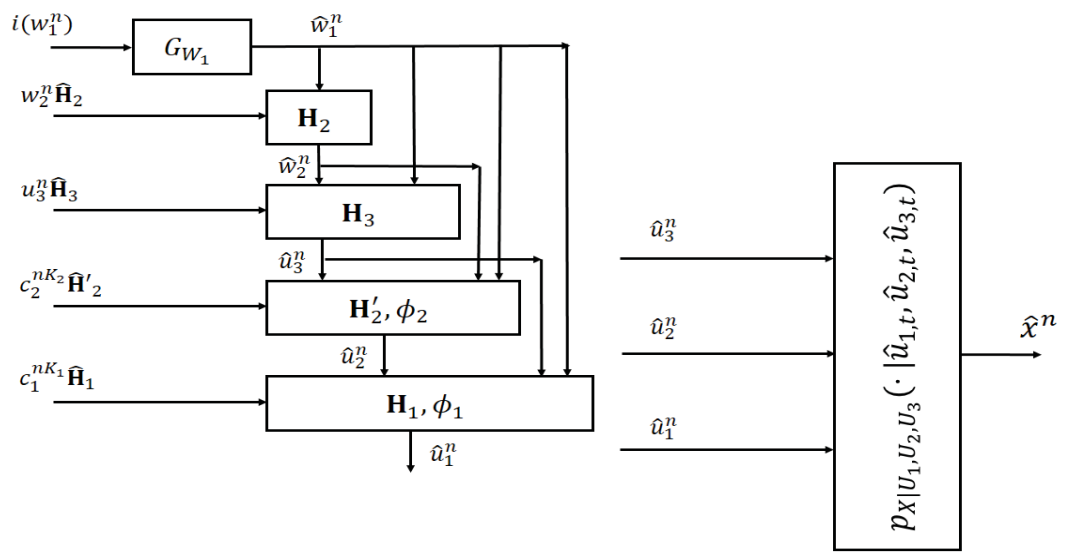

Fig. 3: The proposed successive decoding scheme.

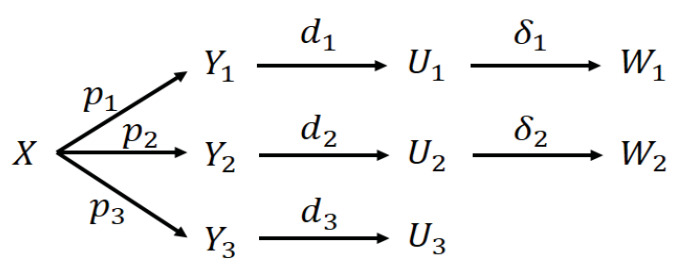

Fig. 4: Joint distribution diagram of the binary CEO problem.

demapping and decoding with $\left(\mathbf{H}_{1}, \phi_{1}\right)$ defining the factor graph and $\left(\hat{w}_{1}^{n}, \hat{u}_{2}^{n}, \hat{u}_{3}^{n}\right)$ serving as the channel output. Set $\hat{u}_{1}^{n}=\hat{w}_{1}^{n} \oplus \phi_{1}\left(\hat{c}_{1}^{n K_{1}}\right)$.

6) Finally, it produces a soft reconstruction $\hat{x}^{n}$ based on $\hat{u}_{1}^{n}, \hat{u}_{2}^{n}$, and $\hat{u}_{3}^{n}($ see $(16))$.

The block diagram of the proposed decoding scheme is depicted in Fig. 3 .

\section{Analysis of the Proposed Coding Scheme}

Now we proceed to specify the sizes of generator matrices and parity-check matrices used in the proposed scheme and other relevant parameters, assuming that $d_{1}, d_{2}, d_{3}$, $\delta_{1}$, and $\delta_{2}$ are given according to the joint distribution of a 3-link binary CEO problem depicted in Fig. 4.

For the LDGM codes $C_{W_{l}}$, as shown in Fig. 2, their generator matrices are of size $m_{l} \times n, l \in \mathcal{I}_{3}$, respectively, where

$$
\begin{aligned}
\frac{m_{i}}{n} & =1-h_{b}\left(d_{i} * \delta_{i}\right)+\epsilon_{i, 1}, \quad i \in \mathcal{I}_{2}, \\
\frac{m_{3}}{n} & =1-h_{b}\left(d_{3}\right)+\epsilon_{3,1} .
\end{aligned}
$$

Furthermore, size of the generator matrix of the LDGM code $C_{i}^{\prime}$ is $M_{i} \times n K_{i}$, for $i \in \mathcal{I}_{2}$. By properly designing these LDGM codes and increasing the block length $n$, one can ensure that

$$
\begin{aligned}
& \mathbb{E}\left(\frac{1}{n} \sum_{j=1}^{n}\left[y_{i, j} \oplus w_{i, j}\right]\right) \approx d_{i} * \delta_{i}, \quad i \in \mathcal{I}_{2}, \\
& \mathbb{E}\left(\frac{1}{n} \sum_{j=1}^{n}\left[y_{l, j} \oplus u_{l, j}\right]\right) \approx d_{l}, \quad \epsilon_{l, 1} \approx 0, \quad l \in \mathcal{I}_{3} .
\end{aligned}
$$

For the LDPC codes shown in Fig. 2, the sizes of their parity-check matrices are given as follows:

$$
\begin{array}{ll}
\mathbf{H}_{1}: n K_{1} \times\left(n K_{1}-M_{1}+k_{1}\right), & \mathbf{H}_{2}: n \times\left(n-m_{2}+k_{2}\right), \\
\mathbf{H}_{2}^{\prime}: n K_{2} \times\left(n K_{2}-M_{2}+k_{2}^{\prime}\right), & \mathbf{H}_{3}: n \times\left(n-m_{3}+k_{3}\right) .
\end{array}
$$

All of the LDPC codes in the decoder performs an SP algorithm. Basically, each SP algorithm is an iterative message-passing algorithm which passes LLR values between variable nodes and check nodes of the LDPC code. In each iteration, we have LLR-updating equations in both variable nodes and check nodes. Generally, there are two types of inputs in this algorithm: (1) Syndrome, (2) Side information. In the SP algorithm, initial LLR values for the variable nodes are calculated based on the joint distribution diagram parameters. For instance, the initial LLR values of the SP algorithm by using parity-check matrix $\mathbf{H}_{3}$ are as follows:

$$
\begin{gathered}
\operatorname{LLR}_{t, 0}=\log \frac{p_{U_{3} \mid W_{1}, W_{2}}\left(0 \mid \hat{w}_{1, t}, \hat{w}_{2, t}\right)}{p_{U_{3} \mid W_{1}, W_{2}}\left(1 \mid \hat{w}_{1, t}, \hat{w}_{2, t}\right)}, \\
\text { for } t \in \mathcal{I}_{n} \quad \text { or } \quad t \in \mathcal{I}_{n K_{i}} .
\end{gathered}
$$

There are four possible cases for the $\operatorname{LLR}_{t, 0}$ based on the values of $\left(\hat{w}_{1, t}, \hat{w}_{2, t}\right) \in \mathbb{B}^{2}$, and all of them can be calculated from the joint distribution diagram.

In the syndrome-decoding part of our proposed scheme, which is implemented by successive SP algorithms, if the optimized degree distributions for the BSC are used with sufficiently long LDPC codes, the Bit Error Rate (BER) for the reconstruction of $\left\{U_{1}, U_{2}, U_{3}\right\}$ can be made very close to zero, i.e., $\mathrm{BER}_{l} \approx 0$ for $l \in \mathcal{I}_{3}$. In such a case, the total distortion of the $l$-th link approximately equals $d_{l}$. In designing procedure of LDPC codes that are employed for the syndrome-generation and the syndrome-decoding, the following relations are considered in their code rates,

$$
\begin{aligned}
& \mathbf{H}_{2}: \frac{m_{2}-k_{2}}{n}=I\left(W_{1} ; W_{2}\right)-\epsilon_{2,3} \\
& =1-h_{b}\left(P_{1} * \delta_{1} * P_{2} * \delta_{2}\right)-\epsilon_{2,3}, \\
& \mathbf{H}_{3}: \frac{m_{3}-k_{3}}{n}=I\left(W_{1}, W_{2} ; U_{3}\right)-\epsilon_{3,3}
\end{aligned}
$$




$$
\begin{aligned}
& =2+h_{b}\left(P_{1} * \delta_{1} * P_{2} * \delta_{2}\right)-H\left(W_{1}, W_{2}, U_{3}\right)-\epsilon_{3,3}, \\
& \mathbf{H}_{2}^{\prime}: \frac{M_{2}-k_{2}^{\prime}}{n K_{2}}=I\left(W_{1}, U_{3} ; U_{2} \mid W_{2}\right)-\epsilon_{2,4} \\
& =H\left(W_{1}, W_{2}, U_{3}\right)-H\left(W_{1}, U_{2}, U_{3}\right)-\epsilon_{2,4}, \\
& \mathbf{H}_{1}: \frac{M_{1}-k_{1}}{n K_{1}}=I\left(U_{2}, U_{3} ; U_{1} \mid W_{1}\right)-\epsilon_{1,4} \\
& =H\left(W_{1}, U_{2}, U_{3}\right)-H\left(U_{1}, U_{2}, U_{3}\right)-\epsilon_{1,4},
\end{aligned}
$$

where $P_{l}=p_{l} * d_{l}$ for $l \in \mathcal{I}_{3}$. Note that, there are four compound LDGM-LDPC codes ${ }^{7}$ in the proposed scheme for a 3-link binary CEO problem. They comprise the LDGM codes $C_{W_{2}}, C_{U_{3}}, C_{1}^{\prime}$, and $C^{\prime}{ }_{2}$, respectively with the LDPC codes of parity-check matrices $\mathbf{H}_{2}, \mathbf{H}_{3}, \mathbf{H}_{1}$, and $\mathbf{H}_{2}^{\prime}$.

\section{Analytical Results}

It is clear that, for the proposed scheme, there is freedom in choosing $\left(d_{1}, \cdots, d_{L}\right)$ and $\left(\delta_{1}, \cdots, \delta_{L}\right)$. The role of $\left(d_{1}, \cdots, d_{L}\right)$ is to specify the dominant face $\mathcal{F}_{\mathrm{CEO}}\left(p_{U_{l} \mid Y_{l}}, l \in\right.$ $\mathcal{I}_{L}$ ) (and consequently the sum rate) while the role of $\left(\delta_{1}, \cdots, \delta_{L}\right)$ is to specify the location of the target rate tuple $\left(R_{1}, \cdots, R_{L}\right)$ on the dominant face. Note that for any $\left(R_{1}, \cdots, R_{L}, D\right) \in \mathcal{R} \mathcal{D}_{\mathrm{CEO}}\left(p_{U_{l} \mid Y_{l}}, l \in \mathcal{I}_{L}\right)$, we have $\sum_{l=1}^{L} R_{l} \geq R_{\mathrm{th}}$ and $D \geq D_{\mathrm{th}}$, where $R_{\mathrm{th}}=I\left(Y_{\mathcal{I}_{L}} ; U_{\mathcal{I}_{L}}\right)$, and $D_{\text {th }}=H\left(X \mid U_{\mathcal{I}_{L}}\right)$. One can interpret $R_{\text {th }}$ and $D_{\text {th }}$ as the minimum achievable sum rate and distortion associated with a given $\left(d_{1}, \cdots, d_{L}\right)$. Therefore, it is natural to choose $\left(d_{1}, \cdots, d_{L}\right)$ that achieves an optimal tradeoff between $R_{\mathrm{th}}$ and $D_{\mathrm{th}}$, which motivates the following definition.

Definition 5: An $L$-tuple $\left(d_{1}^{*}, \cdots, d_{L}^{*}\right)$ is called an optimal $d$-allocation if it is a minimizer of $F$ for a certain $\mu \geq 0$, where

$$
F=D_{\mathrm{th}}+\mu R_{\mathrm{th}}
$$

We shall derive several analytical results surrounding Definition 5. An investigation along this line was initiated in [20] for the case $L=2$.

Note that

$$
\begin{aligned}
R_{\mathrm{th}} & =H\left(U_{\mathcal{I}_{L}}\right)-H\left(U_{\mathcal{I}_{L}} \mid Y_{\mathcal{I}_{L}}\right) \\
& =H\left(U_{\mathcal{I}_{L}}\right)-H\left(Z_{\mathcal{I}_{L}}\right) \\
& =H\left(U_{\mathcal{I}_{L}}\right)-\sum_{l=1}^{L} h_{b}\left(d_{l}\right),
\end{aligned}
$$

and

$$
\begin{aligned}
D_{\mathrm{th}} & =H\left(X, U_{I_{L}}\right)-H\left(U_{I_{L}}\right) \\
& =H(X)+H\left(U_{I_{L}} \mid X\right)-H\left(U_{I_{L}}\right) \\
& =H(X)+H\left(N_{I_{L}} \oplus Z_{I_{L}}\right)-H\left(U_{I_{L}}\right) \\
& =1+\sum_{l=1}^{L} h_{b}\left(P_{l}\right)-H\left(U_{I_{L}}\right)
\end{aligned}
$$

${ }^{7}$ Generally, there is a compound LDGM-LDPC code in the first and the $L$-th link; and there are two compound codes in the $i$-th link, for $i \in[2: L-1]$. Thus, there are totally $2 L-2$ compound codes in an $L$-link case. where $P_{l}=p_{l} * d_{l}, l \in \mathcal{I}_{L}$. Define $Q_{j}=\prod_{l=1}^{L} \eta\left(P_{l}, b_{l}(j)\right)$ for $j \in\left[0: 2^{L}-1\right]$, where $b_{l}(j)$ denotes the $l$-th digit in the binary expansion of $j$, and

$$
\eta\left(P_{l}, b_{l}(j)\right)= \begin{cases}P_{l}, & b_{l}(j)=0 \\ 1-P_{l}, & b_{l}(j)=1 .\end{cases}
$$

For example, when $L=3$, we have

$$
\begin{array}{llrl}
Q_{0} & =P_{1} P_{2} P_{3}, & Q_{4} & =\left(1-P_{1}\right) P_{2} P_{3}, \\
Q_{1} & =P_{1} P_{2}\left(1-P_{3}\right), & Q_{5} & =\left(1-P_{1}\right) P_{2}\left(1-P_{3}\right), \\
Q_{2} & =P_{1}\left(1-P_{2}\right) P_{3}, & Q_{6} & =\left(1-P_{1}\right)\left(1-P_{2}\right) P_{3}, \\
Q_{3} & =P_{1}\left(1-P_{2}\right)\left(1-P_{3}\right), & Q_{7} & =\left(1-P_{1}\right)\left(1-P_{2}\right)\left(1-P_{3}\right) .
\end{array}
$$

It can be verified that

$$
H\left(U_{I_{L}}\right)=-\sum_{j=0}^{2^{L}-1}\left[\frac{Q_{j}+Q_{2^{L}-1-j}}{2}\right] \log \left[\frac{Q_{j}+Q_{2^{L}-1-j}}{2}\right] .
$$

Lemma 1: For the objective function $F$ defined in (28), its minimum value is equal to 1 when $\mu \geq 1$.

Proof: It is clear that $F=1$ when $\left(d_{1}, \cdots, d_{L}\right)=$ $(0.5, \cdots, 0.5)$. Now assume that a certain choice of $\left(d_{1}, \cdots, d_{L}\right)$ gives $F<1$. As a consequence, we have

$$
\mu<\frac{1-D_{\mathrm{th}}}{R_{\mathrm{th}}}=\frac{H\left(U_{I_{L}}\right)-\sum_{l=1}^{L} h_{b}\left(p_{l} * d_{l}\right)}{H\left(U_{I_{L}}\right)-\sum_{l=1}^{L} h_{b}\left(d_{l}\right)} \leq 1,
$$

which is contradictory with the fact that $\mu \geq 1$.

Lemma 2: Let $p_{1} \leq p_{2}$ and $d_{1}>d_{2}$. If $P_{1}=p_{1} * d_{1}$, $P_{2}=p_{2} * d_{2}, P_{1}^{\prime}=p_{1} * d_{2}$, and $P_{2}^{\prime}=p_{2} * d_{1}$, then

$$
\begin{aligned}
P_{1}+P_{2} & >P_{1}^{\prime}+P_{2}^{\prime}, \\
P_{1} P_{2} & >P_{1}^{\prime} P_{2}^{\prime}, \\
2\left[P_{1} P_{2}-P_{1}^{\prime} P_{2}^{\prime}\right] & =\left[P_{1}+P_{2}\right]-\left[P_{1}^{\prime}+P_{2}^{\prime}\right] .
\end{aligned}
$$

Proof: The proof is straightforward.

Lemma 3: Let $p_{1} \leq p_{2} \leq \cdots \leq p_{L}$. If $d_{1}>d_{2}$ in the $L-$ tuple $\left(d_{1}, \cdots, d_{L}\right)$, then by swapping $d_{1}$ and $d_{2}$, the value of $H\left(U_{I_{L}}\right)$ will increase.

Proof: See Appendix A.

Lemma 4: If $p_{1} \leq p_{2} \leq \cdots \leq p_{L}$, then $d_{1}^{*} \leq d_{2}^{*} \leq \cdots \leq$ $d_{L}^{*}$

Proof: Assume that this is not true, and thus there exits $i$ such that $d_{i}^{*}>d_{i+1}^{*}$. We prove that by swapping $d_{i}^{*}$ and $d_{i+1}^{*}$, the objective function $F=D_{\mathrm{th}}+\mu R_{\mathrm{th}}$ will decrease, which is a contradiction. Note that

$$
F=1+\sum_{l=1}^{L} h_{b}\left(p_{l} * d_{l}^{*}\right)-\mu \sum_{l=1}^{L} h_{b}\left(d_{l}^{*}\right)+(\mu-1) H\left(U_{\mathcal{I}_{L}}\right) .
$$

Based on Lemmas 1 and 2, term $(\mu-1) H\left(U_{\mathcal{I}_{L}}\right)$ decreases by swapping $d_{i}^{*}$ and $d_{i+1}^{*}$. Also, the term $-\mu \sum_{l=1}^{L} h_{b}\left(d_{l}^{*}\right)$ clearly remains unchanged by this replacement. Without loss of generality, let us assume $i=1$. Therefore, it is enough to show that

$$
h_{b}\left(p_{1} * d_{2}^{*}\right)+h_{b}\left(p_{2} * d_{1}^{*}\right)<h_{b}\left(p_{1} * d_{1}^{*}\right)+h_{b}\left(p_{2} * d_{2}^{*}\right) .
$$


By defining the following variables $z_{1}$ and $z_{2}$, we have:

$$
\begin{aligned}
& z_{1} \triangleq p_{1} * d_{1}^{*} \Rightarrow p_{1} * d_{2}^{*}<z_{1}<p_{2} * d_{1}^{*}, \\
& z_{2} \triangleq p_{1} * d_{2}^{*}+p_{2} * d_{1}^{*}-p_{1} * d_{1}^{*} \\
& \Rightarrow p_{1} * d_{2}^{*}<z_{2}<p_{2} * d_{2}^{*}<p_{2} * d_{1}^{*}, \\
& \Rightarrow z_{1}+z_{2}=p_{1} * d_{2}^{*}+p_{2} * d_{1}^{*} .
\end{aligned}
$$

Since, $h_{b}(x)$ is a concave function in $x$, from (35)

$$
h_{b}\left(p_{1} * d_{2}^{*}\right)+h_{b}\left(p_{2} * d_{1}^{*}\right)<h_{b}\left(z_{1}\right)+h_{b}\left(z_{2}\right) .
$$

Furthermore, $h_{b}(x)$ is an increasing function in the interval $[0,0.5]$. Thus,

$$
h_{b}\left(z_{1}\right)+h_{b}\left(z_{2}\right)<h_{b}\left(p_{1} * d_{1}^{*}\right)+h_{b}\left(p_{2} * d_{2}^{*}\right) .
$$

From (36) and (37), the inequality (34) is concluded. Hence, the proof is completed.

\section{Numerical Results}

Now we provide some numerical examples of optimal $d$-allocations. Without loss of generality, we assume $p_{1} \leq$ $p_{2} \leq \cdots \leq p_{L}$. It follows by Lemma 4 that $d_{1}^{*} \leq d_{2}^{*} \leq \cdots \leq$ $d_{L}^{*}$ for the resulting optimal $d$-allocation. Obviously, $d_{l}^{*}$ equals 0 for all $l$ 's when $\mu=0$. There exists a $\mu_{0}>0$ such that for $0 \leq \mu<\mu_{0}$, all $L$ links are involved in information sending, i.e., $d_{l}^{*}<0.5$ for $l \in \mathcal{I}_{L}$, while $d_{L}^{*}=0.5$ for $\mu=$ $\mu_{0}$. Therefore, the $L$-th link becomes inactive for $\mu \geq \mu_{0}$. Accordingly, the problem is reduced to an $(L-1)$-link case. By increasing $\mu$, the noisy links are eliminated one-by-one. Finally, it is reduced to the case of $L=2$. We illustrate this phenomenon through the following simple example.

Example 1: Let $L=3$ and $p_{1}=p_{2}=p_{3}=0.1$. Based on the numerical results, if $0 \leq \mu<\mu_{0} \approx 0.3923$, then the straight line $0 \leq d_{1}^{*}=d_{2}^{*}=d_{3}^{*}<0.125$ determines the location of the optimal points. For $\mu_{0} \leq \mu<\mu_{1} \approx 0.42$, we have $d_{1}^{*}=d_{2}^{*} \leq 0.125$ and $0.125<d_{3}^{*}<0.5$. If $\mu=\mu_{1}$, then $d_{1}^{*}=d_{2}^{*}=0.089$ and $d_{3}^{*}=0.5$. Similarly, if $\mu_{1}<\mu<\mu_{2} \approx$ 0.4245 , then $d_{1}^{*}<d_{2}^{*}<0.5$ and $d_{3}^{*}=0.5$. Next, for $\mu_{2} \leq$ $\mu<\mu_{\max }=0.64$, the first link is only involved in sending the information, i.e., $0.023<d_{1}^{*}<0.5$ and $d_{2}^{*}=d_{3}^{*}=0.5$. Finally, $d_{1}^{*}=d_{2}^{*}=d_{3}^{*}=0.5$ for $\mu \geq \mu_{\max }$.

The next example illustrates the sum-rate-distortion tradeoffs under equal $d$-allocation (i.e., $d_{1}=d_{2}=\cdots=$ $\left.d_{L}\right)$.

Example 2: Let $L=3$ and $p_{1}=p_{2}=p_{3}$. The sum-rate distortion curves under equal $d$-allocation are depicted in Fig. 5(a) for various noise parameters. In Fig. 5(b), the sum-rate distortion curves under equal $d$-allocation are shown for the case of $p_{l}=0.25$ with $L=3,5,7,9$.

Example 3: Based on the numerical and the analytical results presented in [20], for a two-link binary CEO problem, the equal allocation, i.e., $d_{1}^{*}=d_{2}^{*}$, is not an optimal $d$ allocation for some values of sum-rate and distortion, even in the case of equal noise parameters $p_{1}=p_{2}$. Here, it is shown that this surprising result is also authentic for the multi-link case. In Fig. 6, the sum-rate distortion curves are shown for some cases. As it is seen, involving all the links does not necessarily provide minimum values of the sum-rate and the distortion.
Example 4: In this example, a 3-link binary CEO problem is considered with almost prominent differences between the values of the noise parameters. As an example, let $p_{1}=0.01, p_{2}=0.1$, and $p_{3}=0.2$. The sum-rate versus the distortion curves are presented in Fig. 7. It is assumed that the binary quantizers in each link are the same, when more than one link are involved in sending the information. Clearly, utilizing low noise links provides better results.

Now we proceed to present some experimental results for the proposed coding scheme. In our implementation, the degree distributions of the LDPC codes are provided in Appendix B; furthermore, the degree distributions of the LDGM codes are designed based on the method proposed in [16], where the degrees of check nodes are regular and those of variable nodes follow a Poisson distribution. The relevant parameters of the proposed scheme are presented in Tables II and III. In particular, each choice of $\left(d_{1}, \cdots, d_{L}\right)$ corresponds to an optimal $d$-allocation. The rate of each encoder is calculated as follows:

$$
\begin{aligned}
R_{1} & =\left(\frac{m_{1}}{n}\right)+\left(I\left(Y_{1} ; U_{1} \mid W_{1}\right)\right) \times \frac{k_{1}}{n} \\
& \approx\left(1-h_{b}\left(d_{1} * \delta_{1}\right)\right)+\left(h_{b}\left(d_{1} * \delta_{1}\right)-h_{b}\left(d_{1}\right)\right) \times \frac{k_{1}}{n}, \\
R_{l} & =\left(\frac{k_{l}}{n}\right)+\left(I\left(Y_{l} ; U_{l} \mid W_{l}\right)\right) \times \frac{k_{l}^{\prime}}{n} \\
& =\left(\frac{m_{l}}{n}-\frac{m_{l}-k_{l}}{n}\right)+\left(I\left(Y_{l} ; U_{l} \mid W_{l}\right)\right) \times \frac{k_{l}^{\prime}}{n} \\
& \approx\left(1-h_{b}\left(d_{l} * \delta_{l}\right)\right)-\left(I\left(W_{1}, \cdots, W_{l-1} ; W_{l}\right)\right) \\
& +\left(h_{b}\left(d_{l} * \delta_{l}\right)-h_{b}\left(d_{l}\right)\right) \times \frac{k_{l}^{\prime}}{n}, \quad l \in[2: L-1], \\
R_{L} & =\left(\frac{k_{L}}{n}\right)=\left(\frac{m_{L}}{n}\right)-\left(\frac{m_{L}-k_{L}}{n}\right) \\
& \approx\left(1-h_{b}\left(d_{L}\right)\right)-\left(I\left(W_{1}, \cdots, W_{L-1} ; U_{L}\right)\right) .
\end{aligned}
$$

Example 5: Consider a 3-link case. Let $p_{1}=0.2$, $p_{2}=0.205$, and $p_{3}=0.21$ as well. For $\mu=0.25$, the optimal $d$-allocation is given by $d_{1}^{*}=0.1, d_{2}^{*}=0.164$, and $d_{3}^{*}=0.377 ;$ as consequence, we have $R_{\mathrm{th}}=0.9091$ and $D_{\text {th }}=0.7243$. The performance of the proposed coding scheme is presented for the corner and the intermediate points separately. The block lengths are equal to $n=10^{4}$, $n=5 \times 10^{4}$, and $n=10^{5}$. First, to achieve a corner point, we set $\delta_{1}=\delta_{2}=0$. However, in order to achieve an intermediate point, any choice of $\delta_{1} \in(0,0.5)$ and $\delta_{2} \in(0,0.5)$ gives a specific intermediate point on the dominant face. In this example, we set $K_{1}=7, K_{2}=6$, $M_{1}=0.22 n K_{1}$, and $M_{2}=0.19 n K_{2}$ for the intermediate point. The results are presented in Table II and Fig. 8. The gap values for the code lengths $n=10^{4}, 5 \times 10^{4}$, and $10^{5}$ are about $0.029,0.023$, and 0.02 , respectively.

Example 6: Consider a 4-link case and let $p_{l}=0.1$ for $l \in \mathcal{I}_{4}$. For $\mu=0.27$, the optimal $d$-allocation is given by $d_{l}^{*}=0.1$, for $l \in \mathcal{I}_{4}$; as a consequence, we have $R_{\mathrm{th}}=1.591$ and $D_{\text {th }}=0.2534$. The block lengths are set to $n=10^{4}$, $n=5 \times 10^{4}$, and $n=10^{5}$. In order to achieve a corner point, we set $\delta_{1}=\delta_{2}=\delta_{3}=0$. However, to achieve an intermediate point, any choice of $\delta_{i} \in(0,0.5)$ for $i \in \mathcal{I}_{3}$, gives a specific intermediate point. In this example, we set 


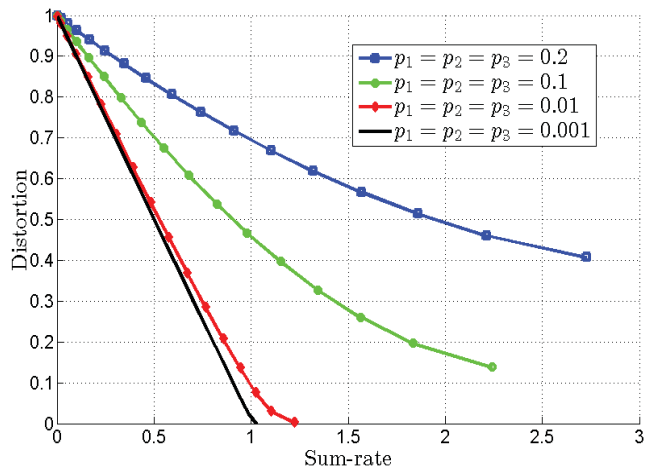

(a) Equal noise parameters with the same quantizer in each link, the number of links is $L=3$.

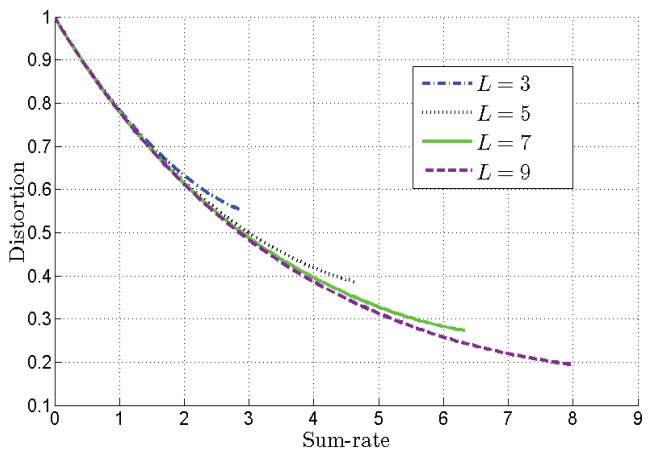

(b) $p_{l}=0.25$ for $l \in \mathcal{I}_{L}$.

Fig. 5: Sum-rate vs. distortion curves under the BSC assumption for the test channels.

TABLE II: PARAMETERS AND NUMERICAL RESULTS OF THE PROPOSED CODING SCHEME, (Example 5).

\begin{tabular}{|c|c|c|c|c|c|c|c|}
\hline$m_{1}, m_{2}, m_{3}$ & $k_{1}, k_{2}, k_{2}^{\prime}, k_{3}$ & $\delta_{1}, \delta_{2}$ & $d_{1}, d_{2}, d_{3}$ & $R_{1}, R_{2}, R_{3}, R$ & $\mathrm{BER}_{1}, \mathrm{BER}_{2}, \mathrm{BER}_{3}, \mathrm{BER}_{4}$ & $D_{\mathrm{em}}$ & Gap \\
\hline$\overline{54400,4000,500}$ & $2-, 3700,-, 500$ & $\overline{0,0}$ & $0.102,0.1669,0.3783$ & $0.54,0.4,0.05,0.99$ & $0.0022,0.0025,-,--$ & 0.7532 & 0.0289 \\
\hline $3300,2100,500$ & $9900,2000,9950,500$ & $0.1024,0.141$ & $0.1036,0.1677,0.3783$ & $0.538,0.3868,0.05,0.9748$ & $0.001,0.0014,0.0014,0.0021$ & 0.759 & 0.0347 \\
\hline $26500,18250,2300$ & $-, 18000,-, 2300$ & 0,0 & $0.1014,0.1658,0.3778$ & $0.53,0.36,0.046,0.936$ & $0.0012,0.0 .0016,-,-$ & 0.7474 & 0.0231 \\
\hline $16000,9500,2300$ & $49500,9500,49750,2300$ & $0.1024,0.141$ & $0.1019,0.166,0.3778$ & $0.5304,0.3677,0.0460,0.9441$ & $0.0009,0.0 .0012,0.001,0.0015$ & 0.7552 & 0.0309 \\
\hline 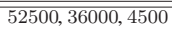 & $\overline{-,, 35000,-, 4500}$ & 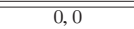 & $0.1009,0.1653,0.3776$ & $0.525,0.35$, & $0.001,0.0$ & 0.7438 & 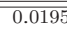 \\
\hline $31500,18000,4500$ & $99000,17500,99500,4500$ & $0.1024,0.141$ & $0.1014,0.1648,0.3776$ & $0.5261,0.3542,0.045,0.9253$ & $0.0006,0.0011,0.0008,0.0014$ & 0.7503 & 0.026 \\
\hline
\end{tabular}

TABLE III: PARAMETERS AND NUMERICAL RESULTS OF THE PROPOSED CODING SCHEME, (Example 6).

\begin{tabular}{|c|c|c|c|c|c|c|c|}
\hline$m_{1 \leq i \leq 3}, m_{4}$ & $k_{1}, k_{2}, k_{2}^{\prime}, k_{3}, k_{3}^{\prime}, k_{4}$ & $\delta_{1}, \delta_{2}, \delta_{3}$ & $d_{1}, d_{2}, d_{3}, d_{4}$ & $R_{1}, R_{2}, R_{3}, R_{4}, R$ & $\mathrm{BER}_{1}, \mathrm{BER}_{2}, \mathrm{BER}_{3}, \mathrm{BER}_{4}, \mathrm{BER}_{5}, \mathrm{BER}_{6}$ & $D_{\mathrm{em}}$ & Gap \\
\hline 5500 & $=, 4400,-, 4000,-, 3600$ & $\overline{0,0,}$ & $\begin{array}{ll}0.1025,0.1028,0.1031, \\
\end{array}$ & $0.55,0.44,0.4,0.36,1$. & 0.4 & 0.2743 & $\overline{02}$ \\
\hline & $9950,430 \mathrm{C}$ & 81,0 & & 441,0 & 5,0.0 & & \\
\hline 2700 &,- 21. & & & & & & $\overline{0.0144}$ \\
\hline 25500 & $49600,20500,49600$, & $.01,0$. & & $0.5343,0$ & $0.0015,0.0018$ & 0.2694 & 0.016 \\
\hline 53000 & $\overline{--, 42000,-, 37}$ & $\overline{0.0,0}$ & (0.1017, & 0.53, & $\overline{0.06}$ & 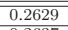 & 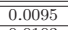 \\
\hline 50500,53000 & $99100,40000,99100,34000,99100,32000$ & $0.01,0.01,0.01$ & $0.1015,0.101,0$ & $0.5293,0.4244,0.3643,0.32,1.638$ & $0.0009,0.0012,0.001,0.00$ & 0.2637 & \\
\hline
\end{tabular}

$K_{i}=9$ and $M_{i}=0.12 n K_{i}, i \in \mathcal{I}_{3}$, for the intermediate point. The results are shown in Table III and Fig. 9. The gap values for the code lengths $n=10^{4}, 5 \times 10^{4}$, and $10^{5}$ are about $0.021,0.015$, and 0.01 , respectively. According to the results of Examples 5 and 6 , by decreasing the noise parameter or increasing the number of links, the gap values from the theoretical bounds are reduced. Moreover, larger block length $n$ causes smaller gap values.

\section{CONClusion}

We have proposed a practical coding scheme for the binary CEO problem under the log-loss criterion based on the idea of quantization splitting. The underlying methodology is in fact quite general and is applicable to the non-binary case as well. It should be emphasized that, to implement the proposed scheme, one needs to first specify the test channel model for each encoder. In general, it is preferable for the system to operate in a mode that corresponds to a certain boundary point of the ratedistortion region. Identifying the boundary-attaining test channel models is an interesting research problem worthy of further investigation.

\section{ApPendix A}

Proof of Lemma 3
Since $H\left(U_{\mathcal{I}_{L}}\right)$ is a function of $P_{l}$ for $l \in \mathcal{I}_{L}$, we shall denote it by $H_{P}\left(P_{1}, \cdots, P_{L}\right)$. It suffices to show that

$$
H_{P}\left(P_{1}^{\prime}, P_{2}^{\prime}, P_{3}, \cdots, P_{L}\right)>H_{P}\left(P_{1}, P_{2}, P_{3}, \cdots, P_{L}\right),
$$

where $P_{1}^{\prime}=p_{1} * d_{2}$ and $P_{2}^{\prime}=p_{2} * d_{1}$. From (30),

$$
H_{P}\left(P_{1}, P_{2}, \cdots, P_{L}\right)=-\sum_{j=0}^{2^{L}-1} q_{j} \log \left(q_{j}\right),
$$

where $q_{j}=\frac{Q_{j}+Q_{2} L_{-1-j}}{2}$. Hence, (39) can be written as follows:

$$
-\sum_{j=0}^{2^{L}-1} q_{j}^{\prime} \log \left(q_{j}^{\prime}\right)>-\sum_{j=0}^{2^{L}-1} q_{j} \log \left(q_{j}\right) .
$$

Partition $\left(q_{j}\right)$ 's and $\left(q_{j}^{\prime}\right)$ 's in some groups with four members as follows:

$$
\begin{aligned}
& q_{a}=\frac{P_{1} P_{2} \Psi+\left(1-P_{1}\right)\left(1-P_{2}\right) \Psi^{\prime}}{2}, \\
& q_{b}=\frac{P_{1}\left(1-P_{2}\right) \Psi+\left(1-P_{1}\right) P_{2} \Psi^{\prime}}{2}, \\
& q_{c}=\frac{\left(1-P_{1}\right) P_{2} \Psi+P_{1}\left(1-P_{2}\right) \Psi^{\prime}}{2}, \\
& q_{d}=\frac{\left(1-P_{1}\right)\left(1-P_{2}\right) \Psi+P_{1} P_{2} \Psi^{\prime}}{2},
\end{aligned}
$$




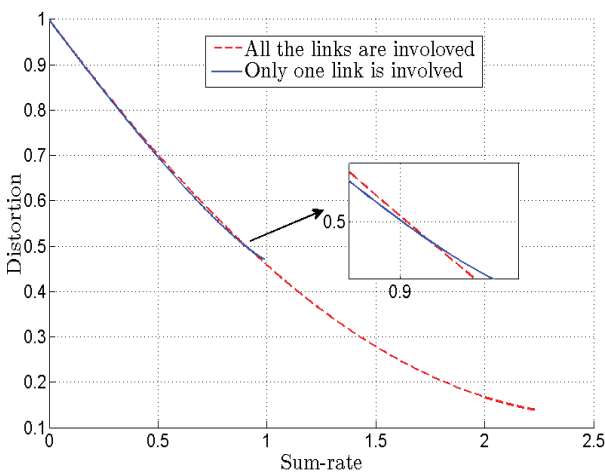

(a)

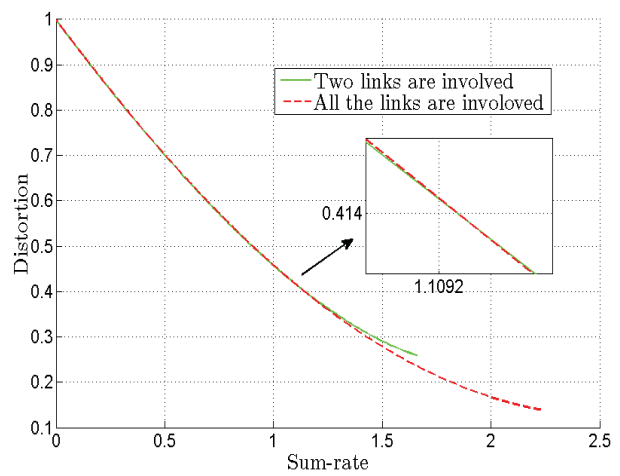

(b)

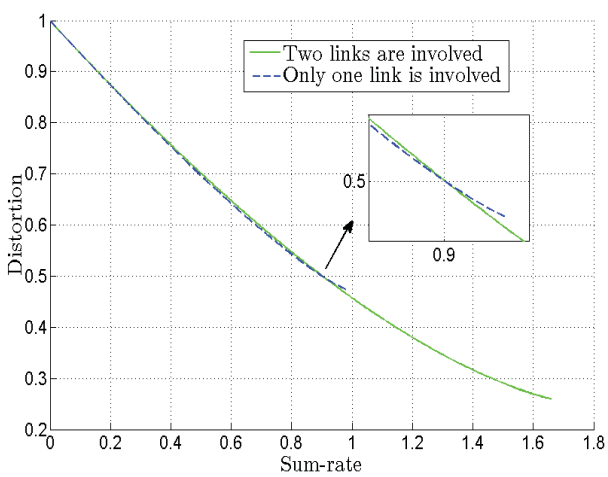

(c)

Fig. 6: Sum-rate vs. distortion curves under the BSC assumption for the test channels. $L=3$ and $p_{1}=p_{2}=p_{3}=0.1$ in different allocation scenarios.

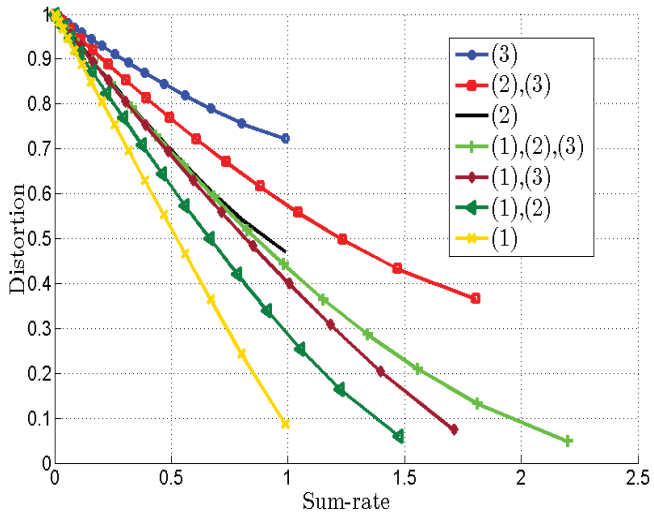

Fig. 7: Sum-rate vs. distortion curves under the BSC assumption for the test channels (Example 4). The number of involved links are given in the legend.

and

$$
\begin{aligned}
& q_{a}^{\prime}=\frac{P_{1}^{\prime} P_{2}^{\prime} \Psi+\left(1-P_{1}^{\prime}\right)\left(1-P_{2}^{\prime}\right) \Psi^{\prime}}{2}, \\
& q_{b}^{\prime}=\frac{P_{1}^{\prime}\left(1-P_{2}^{\prime}\right) \Psi+\left(1-P_{1}^{\prime}\right) P_{2}^{\prime} \Psi^{\prime}}{2}, \\
& q_{c}^{\prime}=\frac{\left(1-P_{1}^{\prime}\right) P_{2}^{\prime} \Psi+P_{1}^{\prime}\left(1-P_{2}^{\prime}\right) \Psi^{\prime}}{2},
\end{aligned}
$$

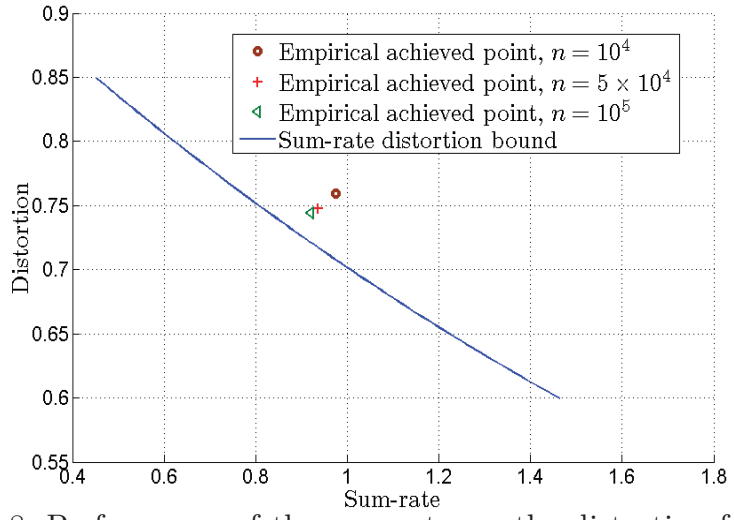

Fig. 8: Performance of the sum-rate vs. the distortion for the implemented codes, (Example 5).

$$
q_{d}^{\prime}=\frac{\left(1-P_{1}^{\prime}\right)\left(1-P_{2}^{\prime}\right) \Psi+P_{1}^{\prime} P_{2}^{\prime} \Psi^{\prime}}{2},
$$

where $\Psi$ is an arbitrary product of $P_{i}$ or $\left(1-P_{i}\right)$, for $i \in$ $[3: L]$, and

$$
\Psi^{\prime}=\frac{P_{3} \cdots P_{L} \times\left(1-P_{3}\right) \cdots\left(1-P_{L}\right)}{\Psi} .
$$

Without loss of generality, it can be assumed that $\Psi \leq$ $\Psi^{\prime}$. Therefore, $q_{a}>q_{d}$ and $q_{a}^{\prime}>q_{d}^{\prime}$. By applying Lemma 


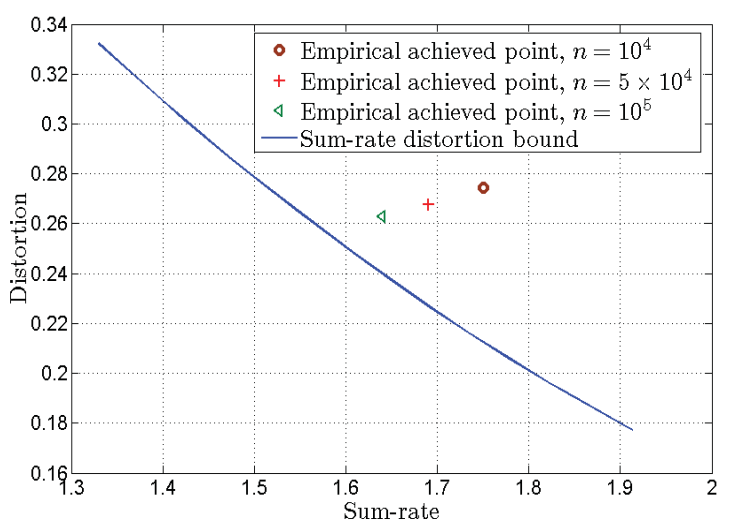

Fig. 9: Performance of the sum-rate vs. distortion for the implemented codes, (Example 6).

2 ,

$$
2\left(q_{a}-q_{a}^{\prime}\right)=
$$

$\Psi\left[P_{1} P_{2}-P_{1}^{\prime} P_{2}^{\prime}\right]+\Psi^{\prime}\left[P_{1} P_{2}-P_{1}-P_{2}-P_{1}^{\prime} P_{2}^{\prime}+P_{1}^{\prime}+P_{2}^{\prime}\right]=$ $\Psi\left[P_{1} P_{2}-P_{1}^{\prime} P_{2}^{\prime}\right]+\Psi^{\prime}[0]>0 \Rightarrow q_{a}>q_{a}^{\prime}$.

Similarly, we can show $q_{d}^{\prime}>q_{d}$. Thus, $q_{a}>q_{a}^{\prime}>q_{d}^{\prime}>q_{d}$. Now consider two following cases:

1) $P_{1} \geq P_{2}$ :

$$
\begin{aligned}
& P_{1} \geq P_{2} \quad \Rightarrow \quad q_{c} \geq q_{b}, \quad q_{c}^{\prime} \geq q_{b}^{\prime} \\
& 2\left(q_{c}-q_{c}^{\prime}\right)=\Psi\left[P_{1}^{\prime} P_{2}^{\prime}-P_{2}^{\prime}+P_{2}-P_{1} P_{2}\right] \\
& +\Psi^{\prime}\left[P_{1}^{\prime} P_{2}^{\prime}-P_{1}^{\prime}+P_{1}-P_{1} P_{2}\right] \\
& >\Psi\left[P_{1}^{\prime} P_{2}^{\prime}-P_{2}^{\prime}+P_{2}-P_{1} P_{2}\right] \\
& +\Psi\left[P_{1}^{\prime} P_{2}^{\prime}-P_{1}^{\prime}+P_{1}-P_{1} P_{2}\right]=0 \Rightarrow q_{c}>q_{c}^{\prime} .
\end{aligned}
$$

Note that in this case, $P_{1}^{\prime} P_{2}^{\prime}-P_{1}^{\prime}+P_{1}-P_{1} P_{2}=$ $\frac{P_{2}^{\prime}-P_{1}^{\prime}+P_{1}-P_{2}}{2} \geq 0$. Similarly, we can show $q_{b}^{\prime}>q_{b}$. Thus, $q_{c}>q_{c}^{\prime}>q_{b}^{\prime}>q_{b}$.

2) $P_{1}<P_{2}$ :

$$
\begin{aligned}
& P_{1}<P_{2} \Rightarrow q_{c}<q_{b}, \quad q_{c}^{\prime}<q_{b}^{\prime} \\
& 2\left(q_{c}-q_{c}^{\prime}\right)=\Psi\left[P_{1}^{\prime} P_{2}^{\prime}-P_{2}^{\prime}+P_{2}-P_{1} P_{2}\right] \\
& +\Psi^{\prime}\left[P_{1}^{\prime} P_{2}^{\prime}-P_{1}^{\prime}+P_{1}-P_{1} P_{2}\right] \\
& <\Psi^{\prime}\left[P_{1}^{\prime} P_{2}^{\prime}-P_{2}^{\prime}+P_{2}-P_{1} P_{2}\right] \\
& +\Psi^{\prime}\left[P_{1}^{\prime} P_{2}^{\prime}-P_{1}^{\prime}+P_{1}-P_{1} P_{2}\right]=0 \Rightarrow q_{c}<q_{c}^{\prime} .
\end{aligned}
$$

Note that in this case, $P_{1}^{\prime} P_{2}^{\prime}-P_{2}^{\prime}+P_{2}-P_{1} P_{2}=$ $\frac{P_{1}^{\prime}-P_{2}^{\prime}+P_{2}-P_{1}}{2} \geq 0$. Similarly, we can show $q_{b}>q_{b}^{\prime}$. Thus, $q_{b}>q_{b}^{\prime}>q_{c}^{\prime}>q_{c}$.

Finally, note that

$$
q_{a}+q_{b}+q_{c}+q_{d}=q_{a}^{\prime}+q_{b}^{\prime}+q_{c}^{\prime}+q_{d}^{\prime}=\frac{\Psi+\Psi^{\prime}}{2} .
$$

Due to the concavity of the function $f(x)=-x \log (x)$, it is concluded that

$$
\begin{aligned}
& -q_{a} \log \left(q_{a}\right)-q_{b} \log \left(q_{b}\right)-q_{c} \log \left(q_{c}\right)-q_{d} \log \left(q_{d}\right) \\
& <-q_{a}^{\prime} \log \left(q_{a}^{\prime}\right)-q_{b}^{\prime} \log \left(q_{b}^{\prime}\right)-q_{c}^{\prime} \log \left(q_{c}^{\prime}\right)-q_{d}^{\prime} \log \left(q_{d}^{\prime}\right) .
\end{aligned}
$$

By doing a summation over all possible values of $\Psi$ in the mentioned 4-tuple groups, (39) is proved.

\section{ApPendix B}

\section{Degree Distributions}

In example 5 , the employed degree distribution of parity-check matrices are as follows, which were obtained based on the degree distributions available in [31].

$\mathbf{H}_{2}: \lambda(x)=0.4145 x+0.1667 x^{2}+0.0571 x^{4}+0.0737 x^{5}+$ $0.0022 x^{8}+0.0118 x^{9}+0.0751 x^{11}+0.0575 x^{19}+0.0063 x^{26}+$ $0.0046 x^{35}+0.0171 x^{43}+0.0443 x^{62}+0.051 x^{82}+0.0165 x^{99}$, and $\rho(x)=0.5 x^{2}+0.5 x^{3}$.

$\mathbf{H}_{3}: \lambda(x)=0.2911 x+0.19 x^{2}+0.0408 x^{4}+0.0874 x^{5}+$ $0.0074 x^{6}+0.1125 x^{7}+0.0925 x^{15}+0.0186 x^{20}+0.124 x^{32}+$ $0.016 x^{39}+0.02 x^{44}$, and $\rho(x)=x^{3}$.

$\mathbf{H}_{1}: \lambda(x)=0.41 x+0.1724 x^{2}+0.0995 x^{4}+0.0546 x^{5}+$ $0.0379 x^{6}+0.0312 x^{10}+0.0288 x^{14}+0.0432 x^{16}+0.0217 x^{20}+$ $0.0385 x^{28}+0.0375 x^{50}+0.0023 x^{52}+0.0158 x^{62}+0.0066 x^{71}$, and $\rho(x)=0.4 x^{2}+0.6 x^{3}$.

$\mathbf{H}_{2}^{\prime}: \lambda(x)=0.3424 x+0.165 x^{2}+0.12 x^{4}+0.0191 x^{5}+$ $0.012 x^{6}+0.1416 x^{10}+0.0211 x^{25}+0.0202 x^{26}+0.0185 x^{34}+$ $0.0429 x^{36}+0.0133 x^{38}+0.0022 x^{39}+0.0104 x^{40}+0.0704 x^{99}$, and $\rho(x)=0.5 x^{2}+0.5 x^{4}$.

In example 6 , the employed degree distribution of parity-check matrices are as follows, which were obtained based on the degree distributions available in [31].

$\mathbf{H}_{2}: \lambda(x)=0.3585 x+0.1664 x^{2}+0.0487 x^{4}+0.1205 x^{5}+$ $0.0006 x^{6}+0.04 x^{10}+0.0744 x^{13}+0.0339 x^{25}+0.0076 x^{30}+$ $0.0564 x^{34}+0.0918 x^{99}$, and $\rho(x)=x^{3}$.

$\mathbf{H}_{3}: \lambda(x)=0.3151 x+0.1902 x^{2}+0.0449 x^{4}+0.1706 x^{6}+$ $0.1405 x^{17}+0.0082 x^{37}+0.044 x^{41}+0.0863 x^{66}$, and $\rho(x)=$ $0.5 x^{3}+0.5 x^{4}$.

$\mathbf{H}_{4}: \lambda(x)=0.292 x+0.174 x^{2}+0.0523 x^{4}+0.0257 x^{5}+$ $0.122 x^{6}+0.0218 x^{8}+0.021 x^{10}+0.0322 x^{14}+0.1128 x^{23}+$ $0.0328 x^{31}+0.0274 x^{44}+0.0048 x^{53}+0.0126 x^{59}+0.0681 x^{99}$, and $\rho(x)=x^{4}$.

$\mathbf{H}_{1}, \mathbf{H}_{2}^{\prime}$, and $\mathbf{H}_{3}^{\prime}: \lambda(x)=0.3037 x+0.1731 x^{2}+0.0671 x^{4}+$ $0.0123 x^{5}+0.1341 x^{6}+0.0314 x^{12}+0.011 x^{14}+0.0257 x^{16}+$ $0.091 x^{19}+0.04 x^{39}+0.0117 x^{51}+0.0189 x^{57}+0.0112 x^{62}+$ $0.0684 x^{76}$, and $\rho(x)=0.4 x^{2}+0.6 x^{4}$.

\section{REFERENCES}

[1] M. Nangir, R. Asvadi, M. Ahmadian-Attari, and J. Chen, "Successive Wyner-Ziv coding for the binary CEO problem under logloss," in 29th Biennial Symposium on Communications (BSC), 2018, pp. 1-5.

[2] J. He, V. Tervo, X. Zhou, X. He, S. Qian, M. Cheng, M. Juntti, and T. Matsumoto, "A tutorial on lossy forwarding cooperative relaying," IEEE Communications Surveys and Tutorials, vol. 21, no. 1, pp. 66-87, Aug. 2018.

[3] Z. Kong, S. A. Aly, and E. Soljanin, "Decentralized coding algorithms for distributed storage in wireless sensor networks," IEEE J. Sel. Areas Commun., vol. 28, no. 2, pp. 261-267, Feb. 2010 .

[4] P. L. Dragotti and M. Gastpar, Distributed source coding: Theory, Algorithms and Applications. Elsevier, 2009.

[5] T. Berger, Z. Zhang, and H. Viswanathan, "The CEO problem," IEEE Trans. Inf. Theory, vol. 42, no. 3, pp. 887-902, May. 1996.

[6] A. Vempaty and L. R. Varshney, "The Non-Regular CEO Problem," IEEE Trans. Inf. Theory, vol. 61, no. 5, pp. 2764-2775, May. 2015.

[7] K. Eswaran and M. Gastpar, "Remote Source Coding under Gaussian Noise : Dueling Roles of Power and Entropy Power," arXiv preprint, arXiv:1805.06515, 2018. 
[8] D. Seo and L. R. Varshney, "The CEO Problem with $r$ th Power of Difference and Logarithmic Distortions," arXiv preprint, arXiv:1812.00903, 2018.

[9] T. A. Courtade and T. Weissman, "Multiterminal source coding under logarithmic loss," IEEE Trans. Inf. Theory, vol. 60, no. 1, pp. 740-761, Jan. 2014.

[10] Y. Zhou, Y. Xu, W. Yu, and J. Chen, "On the optimal fronthaul compression and decoding strategies for uplink cloud radio access networks," IEEE Trans. Inf. Theory, vol. 62, no. 12, pp. $7402-$ 7418, Jan. 2016

[11] R. G. Gallager, Low-Density Parity-Check Codes. Cambridge, MA: MIT Press, 1963.

[12] M. J. Wainwright, E. Maneva, and E. Martinian, "Lossy source compression using low-density generator matrix codes: Analysis and algorithms," IEEE Trans. Inf. Theory, vol. 56, no. 3, pp. 1351-1368, 2010.

[13] T. Filler, "Minimizing embedding impact in steganography using low density codes," Master's thesis, SUNY Binghamton, 2007 .

[14] T. Filler and J. Fridrich, "Binary quantization using belief propagation with decimation over factor graphs of LDGM codes," in Proc. Allerton Conf. Commun., Control, Comput. IEEE, 2007, pp. $1-5$.

[15] M. J. Wainwright and E. Martinian, "Low-density graph codes that are optimal for binning and coding with side information," IEEE Trans. Inf. Theory, vol. 55, no. 3, pp. 1061-1079, March 2009.

[16] M. Nangir, M. Ahmadian-Attari, and R. Asvadi, "Binary Wyner-Ziv code design based on compound LDGM-LDPC structures," IET Commun., vol. 12, no. 4, pp. 375-383, Mar. 2018.

[17] J. Chen and T. Berger, "Successive Wyner-Ziv coding scheme and its application to the quadratic Gaussian CEO problem," IEEE Trans. Inf. Theory, vol. 54, no. 4, pp. 1586-1603, Apr. 2008.

[18] J. Chen, C. Tian, T. Berger, and S. S. Hemami "Multiple description quantization via Gram-Schmidt orthogonalization," IEEE Trans. Inf. Theory, vol. 52, no. 12, pp. 5197-5217, Dec. 2006 .

[19] X. Zhang, J. Chen, S. B. Wicker, and T. Berger, "Successive coding in multiuser information theory," IEEE Trans. Inf. Theory, vol. 53, no. 6 , pp. 2246-2254, Jun. 2007.

[20] M. Nangir, R. Asvadi, M. Ahmadian-Attari, and J. Chen, "Analysis and code design for the binary CEO problem under logarithmic loss," IEEE Trans. Commun., , vol. 66, no. 12, pp. 6003-6014, Dec. 2018.

[21] B. Rimoldi and R. Urbanke, "Asynchronous Slepian-Wolf coding via source-splitting," in Proc. IEEE ISIT., 1997, p. 271.

[22] B. Rimoldi and R. Urbanke, "A rate-splitting approach to the gaussian multiple-access channel," IEEE Trans. Inf. Theory, vol. 42, no. 2, pp. 364-375, 1996.

[23] A. J. Grant, B. Rimoldi, R. L. Urbanke, and P. A. Whiting, "Rate-splitting multiple access for discrete memoryless channels," IEEE Trans. Inf. Theory, vol. 47, no. 3, pp. 873-890, 2001.

[24] J. Wang, J. Chen, L. Zhao, P. Cuff, and H. Permuter, "On the role of the refinement layer in multiple description coding and scalable coding," IEEE Trans. Inf. Theory, vol. 57, no. 3, pp. 1443-1456, Mar. 2011.

[25] A. El Gamal and Y.-H. Kim, Network information theory Cambridge, U.K.: Cambridge Univ. Press, 2011.

[26] R. Gallager, Information Theory and Reliable Communication. New York, NY: John Wiley \& Sons, 1968.

[27] Z. Sun, M. Shao, J. Chen, K. M. Wong, and X. Wu, "Achieving the rate-distortion bound with low-density generator matrix codes," IEEE Trans. Commun., vol. 58, no. 6, pp. 1643-1653, Jun. 2010.

[28] M. Mondelli, S. H. Hassani, and R. L. Urbanke, "How to achieve the capacity of asymmetric channels," IEEE Trans. Inf. Theory, vol. 64 , no. 5 , pp. $3371-3393$, May 2018.

[29] J. Chen, D.-K. He, and A. Jagmohan, "The equivalence between Slepian-Wolf coding and channel coding under density evolution," IEEE Trans. Commun., vol. 57, no. 9, pp. 2534-2540, Sep. 2009

[30] J. Cao, S. Hranilovic, and J. Chen, "Channel capacity and non-uniform signalling for discrete-time Poisson channels," IEEE/OSA J. Opt. Commun. Netw., vol. 5, no. 4, pp. 329-337, Apr. 2013.

[31] D. H. Schonberg, Practical distributed source coding and its application to the compression of encrypted data. PhD thesis, University of California, Berkeley, 2007.

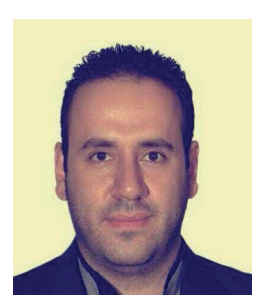

Mahdi Nangir received the B.Sc. degree with the first rank in Electrical Engineering from University of Tabriz and the M.Sc. degree in Communication System Engineering from Sharif University of Technology, Tehran, Iran, in 2010 and 2012, respectively. He received the Ph.D. degree from K. N. Toosi University of Technology, Tehran, Iran, in 2018. He was a finalist of the Mathematics Olympiad and owner of bronze medal in 2005 from Young Scholar Club. He received high ranks in Iranian National Electrical Engineering Student Olympiads of 2009 and 2010. In 2017, he joined McMaster University, Hamilton, Ontario, Canada as a research visiting student. He is now an assistant professor in Faculty of Electrical and Computer Engineering, University of Tabriz, Tabriz, Iran. His research interests include coding and information theory, distributed source coding, and data compression algorithms

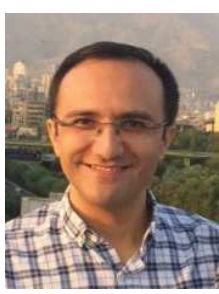

Reza Asvadi (M'11) is an Assistant Professor in Department of Electrical Engineering at Shahid Beheshti University, Tehran, Iran Prior to that, he was a Postdoctoral researcher at the University of Oulu, Oulu, Finland. He received the B.Sc. and the Ph.D. degrees (with the highest honors) in Electrical Engineering from K. N. Toosi University of Technology and the M.Sc. degree from Sharif University of Technology, all in Tehran, Iran. His research interests include coding theory, information theory, signal processing and PHY-layer secrecy. He has published more than 10 journal papers on these topics in prestigious international journals.

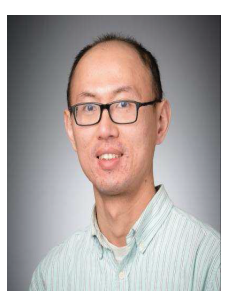

Jun Chen (S'03-M'06-SM'16) received the B.E. degree with honors in communication engineering from Shanghai Jiao Tong University, Shanghai, China, in 2001 and the M.S. and Ph.D. degrees in electrical and computer engineering from Cornell University, Ithaca, NY, in 2004 and 2006, respectively. He was a Postdoctoral Research Associate in the Coordinated Science Laboratory at the University of Illinois at Urbana-Champaign, Urbana, IL, from September 2005 to July 2006, and a Postdoctoral Fellow at the IBM Thomas J. Watson Research Center, Yorktown Heights, NY, from July 2006 to August 2007. Since September 2007 he has been with the Department of Electrical and Computer Engineering at McMaster University, Hamilton, ON, Canada, where he is currently a Professor. His research interests include information theory, machine learning, wireless communications, and signal processing. He held the title of the Barber-Gennum Chair in Information Technology from 2008 to 2013 and the Joseph Ip Distinguished Engineering Fellow from 2016 to 2018. He was a recipient of the Josef Raviv Memorial Postdoctoral Fellowship (2006), the Early Researcher Award from the Province of Ontario (2010), and the IBM Faculty Award (2010). He served as an Associate Editor for the IEEE Transactions on Information Theory from 2014 to 2016. 


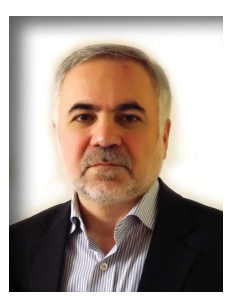

Mahmoud Ahmadian-Attari is Professor and Head of Coding and Cryptography Laboratory (CCL) in the Department of Electrical Engineering at K.N.Toosi University of Technology in Tehran, Iran. He joined K.N.Toosi in 1990. He has previously held positions at the Teacher Training University (currently Kharazmi University), Alzahra University, and the Ministry of Culture and Higher Education (currently Ministry of Science, Research and Technology). He received his M.Eng. degree from the University of Tehran in 1977 and Ph.D. degree from the University of Manchester in 1996, both in Electrical Engineering. His main professional interests are within the areas of Error Correcting Codes, Cryptography, and Secure Communications. He has published some 70 journal papers on these topics, he is the author of the textbook "Error Control Codes in Telecommunication Systems" in Persian (K.N.Toosi Univ. Press, 2013). Since 2013 he has served as member of Communications and signal Processing Chapter of IEEE Iran Section. He is active in conference organization, most recently as the General Chair of the ISCISC'2010 Conference on Information Security and Cryptology, Technical Program Committee in IWCIT'2015. IWCIT'2014, IWCIT'2013 and ISCISC'2011, ISCISC'2012, ISCISC'2013, ISCISC'2014, ISCISC'2015, ISCISC'2016, ISCISC'2017, ISCISC'2018 and 2019.

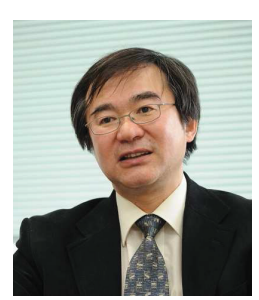

Tad Matsumoto (S'84, M'98, F'10) received his B.S. and M.S. degrees in electrical engineering under the mentorship of Prof. Sin-Ichi Takahashi, and his Ph.D. degree in electrical engineering under the supervision of Prof. Masao Nakagawa, all from Keio University, Yokohama, Japan, in 1978, 1980, and 1991 respectively. He joined Nippon Telegraph and Telephone Corporation (NTT), in 1980, where he was involved in a lot of research and development projects mobile wireless communications systems. In 1992, he transferred to NTT DoCoMo, where he researched on code-division multiple-access techniques for mobile communication systems. In 1994, he transferred to NTT America, where he served as a Senior Technical Advisor of a joint project between NTT and NEXTEL Communications. In 1996, he returned to NTT DoCoMo, where he served as the Head of the Radio Signal Processing Laboratory, until 2001. He researched on adaptive signal processing, multiple-input multiple-output turbo signal detection, interference cancellation, and space-time coding techniques for broadband mobile communications. In 2002, he moved to the University of Oulu, Finland, where he served as a Professor at Centre forWireless Communications. In 2006, he has served as a Visiting Professor with the Ilmenau University of Technology, Ilmenau, Germany, supported by the German MERCATOR Visiting Professorship Program. Since 2007, he has been serving as a Professor with the Japan Advanced Institute of Science and Technology, Japan, while also keeping a cross-appointment position with the University of Oulu (Currently, the cross appointment agreement is frozen). Dr. Matsumoto is a member of the IEICE. He has led a lot of projects supported by the Academy of Finland, European FP7, and the Japan Society for the Promotion of Science and Japanese private companies. He has been appointed as a Finland Distinguished Professor, from 2008 to 2012, supported by Finnish National Technology Agency (Tekes) and Finnish Academy, under which he preserves the rights to participate in and apply for European and Finnish National Projects. $\mathrm{He}$ was a recipient of IEEE VTS Outstanding Service Award, in 2001, Nokia Foundation Visiting Fellow Scholarship Award, in 2002, IEEE Japan Council Award for Distinguished Service to the Society, in 2006, the IEEE Vehicular Technology Society James R. Evans Avant Garde Award, in 2006, Thuringen State Research Award for Advanced Applied Science, in 2006, the 2007 Best Paper Award of the Institute of Electrical, Communication, and Information Engineers of Japan, in 2008, Telecom System Technology Award from the Telecommunications Advancement Foundation, in 2009, IEEE Communication Letters Exemplary Reviewer, in 2011, Nikkei Wireless Japan Award, in 2013, IEEE VTS Recognition for Outstanding Distinguished Lecturer, in 2016, and IEEE TRANSACTIONSON COMMUNICATIONS Exemplary Reviewer, in 2018. He has been serving as an IEEE Vehicular Technology Distinguished Speaker, since 2016 . 